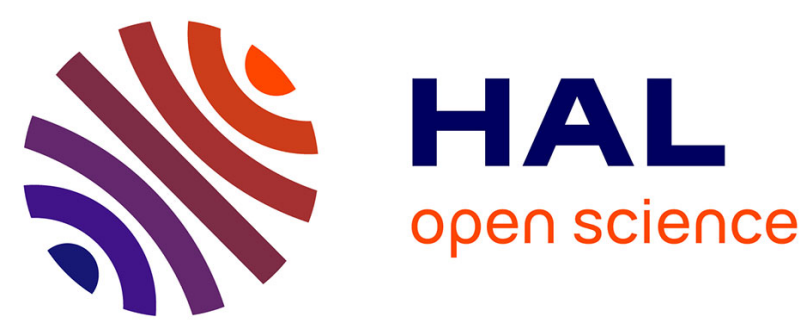

\title{
iAggregator: Multidimensional Relevance Aggregation Based on a Fuzzy Operator
}

\author{
Bilel Moulahi, Lynda Tamine, Sadok Ben Yahia
}

\section{To cite this version:}

Bilel Moulahi, Lynda Tamine, Sadok Ben Yahia. iAggregator: Multidimensional Relevance Aggregation Based on a Fuzzy Operator. Journal of the Association for Information Science and Technology, 2014, vol. 65 ( $\left.n^{\circ} 10\right)$, pp. 2062-2083. 10.1002/asi.23094 . hal-01120754

\section{HAL Id: hal-01120754 \\ https://hal.science/hal-01120754}

Submitted on 26 Feb 2015

HAL is a multi-disciplinary open access archive for the deposit and dissemination of scientific research documents, whether they are published or not. The documents may come from teaching and research institutions in France or abroad, or from public or private research centers.
L'archive ouverte pluridisciplinaire HAL, est destinée au dépôt et à la diffusion de documents scientifiques de niveau recherche, publiés ou non, émanant des établissements d'enseignement et de recherche français ou étrangers, des laboratoires publics ou privés. 


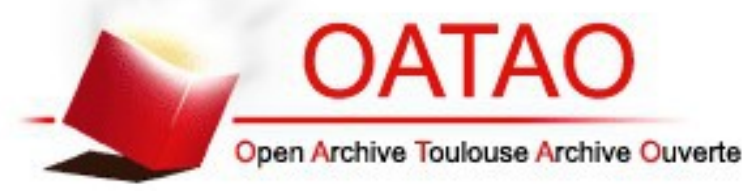

\section{Open Archive TOULOUSE Archive Ouverte (OATAO)}

OATAO is an open access repository that collects the work of Toulouse researchers and makes it freely available over the web where possible.

This is an author-deposited version published in : http://oatao.univ-toulouse.fr/ Eprints ID : 12663

To link to this article : DOI :10.1002/asi.23094

URL : http://dx.doi.org/10.1002/asi.23094

To cite this version : Moulahi, Bilel and Tamine, Lynda and Ben Yahia, Sadok iAggregator: Multidimensional Relevance Aggregation Based on a Fuzzy Operator. (2014) Journal of the Association for Information Science and Technology, vol. 65 ( $\left.\mathrm{n}^{\circ} 10\right)$. pp. 2062-2083. ISSN 2330-1635

Any correspondance concerning this service should be sent to the repository administrator: staff-oatao@,listes-diff.inp-toulouse.fr 


\title{
iAggregator: Multidimensional Relevance Aggregation Based on a Fuzzy Operator
}

\author{
Bilel Moulahi \\ Université Paul Sabatier, Institut de Recherche en Informatique de Toulouse, 118 Route de Narbonne, \\ Toulouse, France, and Université de Tunis El Manar, Faculté des Sciences de Tunis, LIPAH, 2092, Tunis, \\ Tunisie. E-mail: bilel.moulahi@irit.fr \\ Lynda Tamine \\ Université Paul Sabatier, Institut de Recherche en Informatique de Toulouse, 118 Route de Narbonne, \\ Toulouse, France. E-mail: lynda.tamine@irit.fr \\ Sadok Ben Yahia \\ Université de Tunis El Manar, Faculté des Sciences de Tunis, LIPAH, 2092, Tunis, Tunisie; and Institut \\ Mines-TELECOM, TELECOM SudParis, UMR CNRS Samovar, 91011 Evry Cedex, France. \\ E-mail: sadok.benyahia@fst.rnu.tn
}

\begin{abstract}
Recently, an increasing number of information retrieval studies have triggered a resurgence of interest in redefining the algorithmic estimation of relevance, which implies a shift from topical to multidimensional relevance assessment. A key underlying aspect that emerged when addressing this concept is the aggregation of the relevance assessments related to each of the considered dimensions. The most commonly adopted forms of aggregation are based on classical weighted means and linear combination schemes to address this issue. Although some initiatives were recently proposed, none was concerned with considering the inherent dependencies and interactions existing among the relevance criteria, as is the case in many real-life applications. In this article, we present a new fuzzy-based operator, called iAggregator, for multidimensional relevance aggregation. Its main originality, beyond its ability to model interactions between different relevance criteria, lies in its generalization of many classical aggregation functions. To validate our proposal, we apply our operator within a tweet search task. Experiments using a standard benchmark, namely, Text REtrieval Conference Microblog, ${ }^{1}$ emphasize the relevance of our contribution when compared with traditional aggregation schemes. In addition, it outperforms state-of-the-art aggregation operators such as the Scoring and the And prioritized operators as well as some representative learning-to-rank algorithms.
\end{abstract}

${ }^{1}$ https://sites.google.com/site/microblogtrack

\section{Introduction}

Multicriteria aggregation is an issue that has been thoroughly addressed in social choice (Arrow, 1974; Condorcet, 1785; Fishburn, 1972), engineering design (Keeney \& Raiffa, 1993; Neumann \& Morgenstern, 1953), and computer vision applications (Dubois \& Prade, 2004; Torra, 2005), to cite but a few. The multicriteria aggregation arises when for a given task there are several alternatives that have to be ordered with respect to different criteria and we are faced with the problem of combining them to figure out a ranking over the set of alternatives. The need to aggregate several inputs into a single representative output allowed successful applications of aggregation functions to fields as diverse as information retrieval (IR) (Farah \& Vanderpooten, 2007) multiple criteria decision analysis (Grabisch, Kojadinovic, \& Meyer, 2008; Steuer, 1986), data fusion (Ah-Pine, 2008; Vogt \& Cottrell, 1999), and database retrieval (Le Calvè \& Savoy, 2000). In this article, we are more interested in the IR field. Because ranking and relevance are at the heart of IR systems (Hawking, Craswell, Bailey, \& Griffiths, 2001), a great deal of research has triggered a resurgence of interest in revisiting the concept of relevance considering several criteria. In fact, many of the proposed state-of-the-art early IR models rank documents by computing single scores separately with respect to one single objective criterion, rather than considering other relevance dimensions encompassing contextual features with respect to users or documents (Borlund, 2003). This most 
commonly used criterion, which in some applications even becomes a synonym for relevance, is the topical one, namely, subject relevance (Vickery, 1959). It expresses the document's topical overlap with the user's information need, which is solely based on the topicality matching. However, several studies showed that relevance is a multidimensional concept (Borlund, 2003; Saracevic, 2007; Taylor, 2012; Taylor, Cool, Belkin, \& Amadio, 2007) that goes beyond simple topical relevance. Taylor (2012, p. 145) conducted an experimental study and reported that "IR systems must provide a richer set of search criteria beyond topicality."

Furthermore, this multidimensional property is witnessed in many IR applications such as mobile IR (Bouidghaghen et al., 2011; Church \& Smyth, 2008; Cong, Jensen, \& Wu, 2009; Göker \& Myrhaug, 2008), social IR (Becker, Naaman, \& Gravano, 2011; Berardi, Esuli, Marcheggiani, \& Sebastiani, 2011; Chen et al., 2012; Damak, Jabeur, Cabanac, Pinel-Sauvagnat, Tamine, \& Boughanem, 2011; Ounis, Macdonald, \& Soboroff, 2011), and personalized IR (Costa Pereira, Dragoni, \& Pasi, 2009, 2012; Daoud, Tamine, \& Boughanem, 2010; Gauch, Chaffee, \& Pretschner, 2003; Liu, Yu, \& Meng, 2004; Ma, Pant, \& Sheng, 2007). In a mobile IR setting, users usually search for information while moving. The goal of any IR system addressing this issue is to tailor the search results to the user's needs according to several contextual criteria such as location, time, and the user's interests to deliver the information that better addresses the user's situation in spatiotemporal applications. Whereas personalized IR approaches consider user preferences as the main relevance criteria, social IR considers the user's community rather than just the individual as the basic clue for relevance computation. The latter problem is addressed in many settings by involving some significant features regarding the search task at hand. For instance, the tweet search task is driven by a variety of criteria such as authority, topicality, and recency of tweets (Chen et al., 2012; Duan, Jiang, Qin, Zhou, \& Shum, 2010).

Thus, the main challenge that arises is to find a suitable aggregation scheme to combine the single scores related to single criteria evaluations into a global score of documents representing the overall relevance estimate. We notice that despite the overwhelming number of publications that highlighted the multidimensional nature of relevance and the wide range of aggregation operators that have been proposed in the literature, the multidimensional relevance aggregation problem in IR has not attracted the attention it deserves (Costa Pereira et al., 2009, 2012). The most widely used forms of aggregation are the weighted sum and its variations as well as linear combination mechanisms because of their simplicity (Damak et al., 2011; Larkey, Connell, \& Callan, 2000; Si \& Callan, 2002; Vogt \& Cottrell, 1999; Wei et al., 2011). However, as stated by Costa Pereira et al. (2009), the major inconvenience of these works is that the criteria are combined in a linear model independently of the user's preferences on the relevance dimensions. Furthermore, in addition to their inability to model multiple users' preferences, these operators are not suitable for the aggregation of interacting criteria because it requires them to act independently.

In this article, we are concerned with the application of a more sophisticated operator, already of use in other fields, to handle the multidimensional relevance aggregation problem in IR. This operator, named the Choquet integral (Choquet, 1953; Grabisch, 1995), is a successful paradigm in multicriteria decision-making (MCDM) problems (Grabisch \& Labreuche, 2010). The Choquet integral generalizes many other aggregation operators (Grabisch, 1995) such as the weighted mean (Wam) and the Owa operator (Yager, 1988). As far as we know, the Choquet integral is not widely used in the IR realm, the present work involving the combination of documents relevance estimates is the first insight into this area. From a theoretical perspective, the Choquet operator exhibits a number of properties that appear to be appealing from an IR point of view. It allows for modeling interactions between several criteria, which are prominent among relevance criteria and can be undesirable phenomena in some IR applications. Interestingly, the proposed aggregation model is general and may be applied to any set of criteria. The main contributions of this article are twofold:

1. We introduce a general multicriteria aggregation approach, namely, iAggregator, based on a well-studied and theoretically justified mathematically aggregation operator, for multidimensional relevance aggregation in the IR domain. Thus, we survey a problem that has not attracted sufficient attention in the literature. We specifically model the multicriteria relevance aggregation within dependent and interacting criteria.

2. We apply and experiment iAggregator to evaluating multicriteria relevance aggregation in a social IR setting, more particularly, on a tweet search task (Ounis et al., 2011; Ounis, Macdonald, \& Soboroff, 2012), where the jointly considered criteria are topicality, recency, and authority.

The remainder of the article is organized as follows: Multidimensional Relevance Aggregation in IR reviews related work on multidimensional relevance aggregation, gives an overview of the learning-to-rank problem for IR, and describes our motivations. We provide, later in the Background: Aggregation in Decision-Making Problems section, a critical overview of the aggregation problem in the MCDM area and specify the problem within an IR task. Our proposal for a multidimensional relevance estimation with the discrete Choquet integral is presented in the iAggregator: A Multidimensional Relevance Aggregation Operator Using the Choquet Integral section. The Experimental Evaluation Setting section describes the experimental setting within a tweet search task. In the Results and Discussion section, we present and discuss the obtained results. The Conclusion section wraps up the article and outlines future work. 


\section{Multidimensional Relevance Aggregation in IR}

In this section, we present a review of related work on multidimensional relevance followed by a synthesis of works dealing with aggregation operators used for that purpose, as well as learning-to-rank approaches.

\section{Relevance in IR: A Multidimensional Concept}

As pointed out by many key articles in the literature (Barry, 1994; Cosijn \& Ingwersen, 2000; Mizzaro, 1998; Saracevic, 2000; Saracevic, Rothenberg, \& Stephan, 1974; Schamber, 1991), relevance is a complex subject and a challenge that has received steady attention in IR studies during the past 2 decades. Whereas early research favored a topical perspective on relevance, more recent research has paid attention to it from various points of view (Borlund, 2003; Taylor, 2012; Taylor et al., 2007), which implies a shift from topical relevance to multidimensional relevance. The great number of contributions devoted to analyzing the multidimensional concept of relevance has led to identifying many types and facets of relevance, such as cognitive and situational relevance, in addition to algorithmic and topical ones. Table 1 gives an overview of these studies.

The studies of Cuadra and Katter (1967) and Rees and Schultz (1967) investigated the factors that may affect relevance, and identified about 40 possible variables that could influence the users' relevance judgments. Cooper (1973) pointed out in an informal work that many factual features based on documents' properties may be included. Cooper distinguishes between "logical relevance" or "topicality" (relevance concerning the topical component) and "utility" (relevance concerning the three components),

TABLE 1. Synthetic overview of empirical studies emphasizing the multidimensional aspect of relevance concept in IR.

\begin{tabular}{|c|c|}
\hline Main references & Studied relevance criteria \\
\hline $\begin{array}{l}\text { Cuadra and Katter (1967) and } \\
\text { Rees and Schultz (1967) }\end{array}$ & $\begin{array}{l}40 \text { criteria including style and level of } \\
\text { difficulty of the document }\end{array}$ \\
\hline Cooper (1973) & $\begin{array}{l}\text { Novelty, informativeness, credibility, } \\
\text { importance, clarity, positive/negative } \\
\text { factors }\end{array}$ \\
\hline Taylor (1986) & $\begin{array}{l}\text { Ease of use, noise reduction, quality, } \\
\text { adaptability, time saving, cost saving }\end{array}$ \\
\hline Schamber (1991) & $\begin{array}{l}10 \text { criteria (three categories: } \\
\text { information, source, presentation) }\end{array}$ \\
\hline Su $(1992,1994)$ & $\begin{array}{l}20 \text { measures (groups: success, efficiency, } \\
\text { utility, user satisfaction) }\end{array}$ \\
\hline Barry (1994) & $\begin{array}{l}24 \text { criteria grouped into seven broad } \\
\text { groups }\end{array}$ \\
\hline Saracevic (1996) & $\begin{array}{l}\text { Topical, algorithmic, cognitive, } \\
\text { situational, motivational/affective } \\
\text { relevance }\end{array}$ \\
\hline Mizzaro (1998) & $\begin{array}{l}\text { Information resources, user problem, } \\
\text { time, components }\end{array}$ \\
\hline Cosijn and Ingwersen (2000) & $\begin{array}{l}\text { Topical, cognitive/pertinence, } \\
\text { situational, sociocognitive }\end{array}$ \\
\hline Borlund (2003) & Topical, cognitive, situational \\
\hline
\end{tabular}

among which are accuracy, credibility, and recency, and assumes that these criteria could impact relevance judgments. In the same context, Barry (1994) claimed that the relevance is a multidimensional concept and cannot be derived from a single relevance criterion. She performed an exploratory study in which she identified 23 categories of relevance. These categories embody numerous criteria that may be applied to documents' content as well as to any aspect of documents such as contextual factors (e.g., the user situation and environmental effects) or quality of the document source (e.g., authority and reputation). Cosijn and Ingwersen (2000) developed a table of manifestations and attributes for relevance where manifestations consist of topical, cognitive, situational, and sociocognitive attributes. In Borlund (2003), the authors emphasize three relevance dimensions: topical, cognitive, and situational. More specifically, it has been shown that the multidimensionality of relevance can be viewed with reference to different conceptions of relevance such as "the classes, types, criteria, degrees, and levels of relevance" (Borlund, 2003, p. 923). Borlund outlines the different conceptions of the multidimensionality of relevance, as well as the inherent aspect of dynamic relevance. Accordingly, in Saracevic (2007, p. 2132), it has been demonstrated that "topicality plays an important, but not at all an exclusive, role in relevance inferences by people. A number of other relevance clues or attributes, enter into relevance inferences"; these criteria affect the user's perception of relevance and interact with topicality as judgments are made.

Roughly speaking, regarding the research focus of early studies on the use of relevance, we can distinguish between two main categories of approaches. In the first category (Cooper, 1971; Harter, 1992; Vickery, 1959), authors consider topicality as the basis of relevance and assume that all the other criteria are topic-dependent. In contrast, other approaches in the same category, mainly involved in IR applications, adopt the idea that there are many different criteria beyond topicality that may influence the user's perception of relevance. However, they did not investigate the design of aggregation functions, and thus used basic aggregation operators such as the arithmetic mean $(\mathrm{Am})$ and the weighted sum. Unlike the previously cited studies, the second category of contributions (Costa Pereira et al., 2009, 2012; Gerani, Zhai, \& Crestani, 2012) aims at designing general theoretical frameworks of relevance aggregation regardless of the application at hand. This line of research did not receive the attention that it deserves, especially in the IR field. Our contribution attempts to fill this gap, by proposing a general flexible aggregation mechanism based on the well-studied and mathematically justified Choquet integral function.

\section{Relevance Aggregation in IR}

In this section, we review the research contributions dealing with IR applications such as mobile IR, personalized IR, social IR, and geographic IR that make use of 
TABLE 2. Synthetic overview of works involving relevance aggregation in IR tasks.

\begin{tabular}{|c|c|c|c|}
\hline IR task & Main references & Used relevance criteria & Aggregation operators \\
\hline Mobile IR & $\begin{array}{l}\text { Göker and Myrhaug (2008); Church and Smyth } \\
\text { (2008); Cong et al. (2009); Hattori et al. (2007); } \\
\text { Cheverst, Davies, Mitchell, Friday, and Efstratiou } \\
\text { (2000); Schilit et al. (2003); Yau, Liu, Huang, and } \\
\text { Yao (2003); Cantera et al. (2008) }\end{array}$ & $\begin{array}{l}\text { Topicality, user interests, user's } \\
\text { location, time, social features }\end{array}$ & Linear combination mechanism \\
\hline Personalized IR & $\begin{array}{l}\text { Gauch et al. (2003); Daoud et al. (2010); Liu et al. } \\
\text { (2004); Ma et al. (2007); Sieg et al. (2007) }\end{array}$ & $\begin{array}{l}\text { Aboutness, coverage, } \\
\text { appropriateness, reliability, } \\
\text { user interests }\end{array}$ & $\begin{array}{l}\text { Linear combination mechanism: } \\
\text { summation of partial relevance } \\
\text { scores, factor product }\end{array}$ \\
\hline Social IR & $\begin{array}{l}\text { Becker et al. (2011); Metzler and Cai (2011); Damak } \\
\text { et al. (2011); Berardi et al. (2011); Ben Jabeur et al. } \\
\text { (2010); Chen et al. (2012); Smith et al. (2008); } \\
\text { Leung et al. (2006) }\end{array}$ & $\begin{array}{l}\text { Content features, Twitter } \\
\text { features, author features; time }\end{array}$ & $\begin{array}{l}\text { Linear combination mechanism: } \\
\text { summation of partial relevance } \\
\text { scores, factor product }\end{array}$ \\
\hline Geographic IR & $\begin{array}{l}\text { Mata and Claramunt (2011); Kishida (2010); Daoud } \\
\text { and Huang (2013) }\end{array}$ & $\begin{array}{l}\text { Content, time, geographic } \\
\text { location, proximity }\end{array}$ & $\begin{array}{l}\text { Linear combination mechanism: } \\
\text { summation of partial relevance } \\
\text { scores }\end{array}$ \\
\hline
\end{tabular}

aggregation operators to compute a global relevance score. In fact, most of the proposed approaches deal with classical aggregation mechanisms, without having a research focus on the modeling of general multicriteria aggregation functions to combine all of the considered criteria. Second, we synthesize works that, in contrast, have specifically a research focus on the design of appropriate combination operators, to support ranking functions in IR, regardless of any application.

Applying basic relevance aggregation operators in IR applications. The application of relevance aggregation is crucially important in many IR applications. It has been experienced without being the research focus in mobile IR (Church \& Smyth, 2008; Cong et al., 2009; Göker \& Myrhaug, 2008), personalized IR (Daoud et al., 2010; Gauch et al., 2003), social IR (Becker et al., 2011; Berardi et al., 2011; Chen et al., 2012; Damak et al., 2011; Ounis et al., 2011), and geographic IR (Daoud \& Huang, 2013; Kishida, 2010; Mata \& Claramunt, 2011). The approaches that have been proposed are mostly based on linear combination mechanisms. Indeed, the main research subject of these works is the simple combination of individual relevance scores in one given IR setting. We provide in Table 2 a synthetic overview of the main IR tasks involving multidimensional relevance aggregation. For each of these tasks, we cite the main research contributions, we give the used relevance criteria, and then we mention the exploited aggregation operator.

In mobile IR settings, Göker and Myrhaug (2008) used both time and location criteria through a linear combination operator to compute the global documents' scores. Cong et al. (2009) proposed an IR model based on a user's location and a topical relevance dimension in which the documents are ranked through a simple linear combination mechanism of both considered criteria.

Yau et al. (2003) combined situation-based adaptation and profile-based personalization into the IR model. A situation is a set of past context attributes and/or actions such as location, time, light, and device, among others. A user profile includes a usage history and general interests that have been automatically learned using a modified naive Bayesian classifier. Cantera et al. (2008) proposed to use the multiplicative competitive interaction (MCI) model for combining topical scores of documents, the geographic location, and the user's interests in a mobile context. The general expression of utility of a document in the MCI model is given by a linear combination of the individual scores. The considered relevance contextual criteria are mainly the location, the context of the used mobile device, combined with text documents scores.

In personalized IR settings, several works, such as Daoud et al. (2010), Gauch et al. (2003), and Sieg et al. (2007), proposed a combination model of original scores and personalized scores of documents computed according to their similarity to the user's profile represented through his or her interests. The aggregation method is the linear combination of the considered criteria. More precisely, the authors compute the overall relevance score of a document as a linear combination of the personalized score obtained and the original one computed with respect to the topical relevance criterion.

In social IR settings, it is notable that a wide range of research has been proposed in the context of the Text REtrieval Conference (TREC) 2011 and 2012 Microblog Tracks. The majority of the proposed approaches in this area are based on linear combination strategies (Lcs) of relevance criteria. Berardi et al. (2011) focused on the problem of retrieval and ranking in Twitter and proposed an IR system called CipCipPy for that purpose. The authors explored the use of text quality ranking measures to filter out of vocabulary tweets, as well as the use of information contained in hashtags and linked content. The individual scores are then combined through a simple linear combination mechanism. Damak et al. (2011) proposed two tweet search models integrating several features. The first one is 
based on content features (e.g., tweet popularity, tweet length), Twitter features (e.g., URL presence/frequency, hashtag), and author features (e.g., number of tweets/ mentions). For the computation of the final score involving these criteria, the authors adopted an Lcs. Metzler and Cai (2011) proposed a learning-to-rank approach taking into account a textual similarity to the query, a time difference between a tweet and a query, as well as some tweet content features such as the URL presence, the hashtag existence, the tweet length, and the percentage of words out of vocabulary.

The combination of geospatial and temporal criteria in geographic IR has been shown to have significant improvements over traditional search engines (Daoud \& Huang, 2013; Kishida, 2010; Mata \& Claramunt, 2011). For instance, Daoud and Huang (2013) propose a geotemporal retrieval strategy that models and exploits geotemporal context-dependent evidence extracted from pseudorelevant feedback documents. The final score of the document is based on combining the content-based score, the temporal score, the geographic score, and the proximity score using a linear combination operator.

Designing specific relevance aggregation operators. To the best of our knowledge, despite the attention paid to the multidimensional property of relevance, as highlighted earlier (see Relevance in IR: A Multidimensional Concept section), only a few recent works have focused on the design of appropriate combination operators to support multidimensional relevance-based ranking functions in IR. Among these studies is the work of Costa Pereira et al. (2009, 2012), in which the authors proposed a multidimensional representation of relevance and suggested a prioritized aggregation scheme based on two prioritized aggregation operators, namely, And and Scoring. This prioritization models a situation where the weight of a less important criterion is proportional to the satisfaction degree of more important criteria. The authors made use of four criteria in a personalized IR setting: aboutness, coverage, appropriateness, and reliability. Bouidghaghen et al. (2011) suggested a multicriteria relevance model, but on a mobile IR setting, based on three dimensions of relevance: topic, interest, and location. To aggregate these relevance criteria, the authors made use of the two previously cited "prioritized operators" (Costa Pereira et al., 2009), defining a priority order over the set of relevance dimensions. Palacio, Cabanac, Sallaberry, and Hubert (2010) considered a geographic IR system involving three relevance dimensions: spatial, temporal, and topical information. The proposed system combines the results of three criteria with Comb* (Fox \& Shaw, 1994) aggregation functions. Gerani et al. (2012) proposed a multicriteria relevancebased method that allows generating a global score that does not necessarily require that the individual scores, which have to be combined, be comparable. The authors rely on the alternating conditional expectation algorithm (Breiman \& Friedman, 1985) and the BoxCox (Box \& Cox,
1964) model to analyze the incomparability problem and perform a score transformation whenever it is necessary. As an IR application, the authors consider a blog opinion IR setting. More recently, Eickhoff, Vries, and Collins-Thompson (2013) introduced a copula-based method for combining multidimensional relevance estimates. The authors model multivariate document relevance scores based on a number of document quality criteria and show that copulas are able to model complex multidimensional dependencies between these relevance criteria. Their approach has been evaluated within three IR tasks for multidimensional relevance aggregation: opinionated blogs retrieval, personalized social bookmarking, and childfriendly web search. The authors tested the proposed copula-based approach against the product and sum baselines, as well as the linear combinations scheme, and showed that it outperforms these three baselines. Thereafter, they investigated the usefulness of the approach in the score fusion problem relying on copula-based extensions of the two popular score fusion schemes CombSUM and CombMNZ (Fox \& Shaw, 1994).

Many other studies dealing with rank aggregation also have been proposed (Dwork, Kumar, Naor, \& Sivakumar, 2001; Wei, Li, \& Liu, 2010). The rank aggregation task that is encountered in many situations such as metasearch (Akritidis, Katsaros, \& Bozanis, 2011; Aslam \& Montague, 2001) consists of computing a consensus ranking given the individual ranking preferences of several judges (Renda \& Straccia, 2003). Given the ranked lists of documents returned by multiple search engines in response to a given query, the problem of metasearch is to combine these lists in a way that optimizes the performance of the combination (Aslam \& Montague, 2001). These ranking fusion methods can be classified based on whether they rely on the scores or the ranks. In fact, the difference between multidimensional relevance aggregation and rank aggregation is that aggregation occurs without dealing with the multidimensional nature of relevance or the criteria used for searching. These ranking functions use different methods in querying, but in most cases, they are based on the topical criterion or topical-dependent factors despite the different used sources of evidence. For instance, Farah and Vanderpooten $(2007,2008)$ proposed a multicriteria framework for rank aggregation using a decision rule-based mechanism operating with the multidimensional property of the topical criterion. Among these dimensions, we cite, for example, the frequency, document length, and prominence.

Learning to rank for IR. Based on machine-learning algorithms, learning-to-rank methods have been widely used in IR to combine multiple document features for the purpose of optimizing document rankings. The features commonly include query-dependent measures such as BM25 scores or query-independent ones such as PageRank importance. Given a training set of queries and the associated ground truth containing document labels (relevant, irrelevant), the 
objective is to optimize a loss function that maps the document feature-based vector onto the most accurate ranking score. Learning-to-rank approaches fall into three categories: pointwise, pairwise, and listwise (Liu, 2009). In the pointwise approach, regression-based algorithms, classification-based algorithms, and ordinal regressionbased algorithms are used to predict relevance scores. The main idea behind the well-known learning-to-rank algorithms that fall into the pairwaise approach, such as RankSVM (Joachims, 2006) and RankNet (Burges et al., 2005), is the optimization of document pairs preference orderings based on a loss function. Listwise learning-to-rank methods straightforwardly represent the ranking task for IR because they minimize a loss function corresponding to standard IR evaluation measures, considering a ranked list of documents as input.

Intuitively speaking, the multidimensional relevance aggregation problem can be tackled by learning-to-rank methods where the features belong to different relevance dimensions. However, although this community has significant expertise in estimating topical document relevance and other additional criteria, the commonly applied combination schemes ignore the problem of modeling complex, multidimension dependencies. In practice, sophisticated learningto-rank techniques tend to offer only limited insight about why they were weighted highly for relevance (Eickhoff et al., 2013). Indeed, these methods do not explore the relevance dimension level within an IR task, and thus do not allow insight into how to consider importance and interaction between groups of features mapped to different relevance dimensions as stated by the aforementioned studies (Borlund, 2003; Saracevic, 2007). Through the fuzzy measure, our Choquet-based aggregation approach is able to model many interactions between criteria and leads to results that are human interpretable. As we previously stated, thanks to the interaction and importance indices, our method offers qualitative understanding of the resulting model.

\section{Contribution and Motivations}

Although many of the proposed approaches perform effectively in some IR applications, they are not effective in real-life applications because the user's needs involve preferences that lead to several relevance criteria that usually interact with each other. In practice, this problem is usually avoided by considering independent criteria (Cong et al., 2009; Göker \& Myrhaug, 2008). Nevertheless, other works (Carterette, Kumar, Rao, \& Zhu, 2011; Eickhoff et al., 2013; Saracevic, 2007; Wolfe \& Zhang, 2010) have shown that relevance criteria usually interact. For instance, Carterette et al. have proved through an empirical study in a tweet search task the existence of a positive correlation between recency and topical relevance criteria.

Moreover, classical aggregation operators are assumed to hold the additive property that can be effective and convenient in some applications, but can also be somewhat inadequate in many real-life IR tasks. For example, consider the relevance assessment of two documents, $D_{1}$ and $D_{2}$, with respect to two relevance criteria. Then, assume that $D_{1}$, equivalently satisfied with respect to both criteria, is preferred to $D_{2}$, for which the global score is biased by one criterion. Actually, this problem can be dealt with by using an averaging operator such as the weighted sum, but this does not give any way of preferring $D_{1}$ over $D_{2}$ if we consider that the latter have apparently the same global relevance scores. Clearly, this preference needs to trade off both relevance criteria appropriately. This becomes particularly challenging if we consider that a low score obtained on a given criterion can be a serious reason for discounting a document. Although some initiatives were recently proposed (Costa Pereira et al., 2012; Gerani et al., 2012), none was concerned with the interactions existing among the relevance criteria, as is the case in many real-life applications. The following example (inspired by Grabisch, 1995) sketches the impact of dependencies between correlated criteria on the global aggregated score.

Example. Consider the problem of estimating the relevance scores of a subset of documents with respect to three relevance criteria: topicality, authority, and popularity. Suppose that an averaging aggregation operator is used to evaluate these scores and assume that the first criterion is more important than the other two; that is, the weights could be $0.4,0.3$, and 0.3 , respectively. Clearly, authority and popularity criteria may interact because, usually, documents published by influential authors are potentially popular and vice versa. Therefore, because these two criteria may present some degree of redundancy, the global evaluation will be overestimated (underestimated) for popular (nonpopular) documents published by influential authors (uninfluential). Moreover, if we deal with a classical aggregation method such as the Wam, the documents scores with respect to these redundant relevance criteria will be double counted. This can be easily tackled by using a suitable fuzzy measure, where a negative interaction between the criteria authority and popularity is modeled to absorb the bias effect of these redundant criteria.

Consider again the three relevance criteria and suppose that one requires that the satisfaction of only one criterion produces almost the same effect as the satisfaction of both. For example, it is important that documents should be either popular or published by potential (or influential) users. Of course, it is better that they would be relevant with respect to both criteria. Clearly, such a behavior cannot be expressed by a classical aggregation method. In this situation, the importance of the pair is close to that of the single criterion, even within the presence of the other criteria. This condition could be easily expressed using a fuzzy measure, by modeling again a negative interaction. Alternatively, one can require that the satisfaction of only 
one criterion produces a very weak effect compared with the satisfaction of both. Then we speak about a positive interaction, where documents that are equivalently satisfied by all the sets of criteria should be preferred to those that are overestimated by one single relevance criterion.

To address these challenges, we propose to investigate the combination of general-level relevance dimensions using a fuzzy-based aggregation operator. More oriented to the specific problem of relevance aggregation, our method is able to address the property of interaction between dimensions by modeling an integral aggregation function, namely, the Choquet integral, with respect to a fuzzy measure expressing both their individual and joint importance. This aggregation method has the advantage of facilitating the task of interpreting the interactions between the relevance criteria with readily available interpretations via the Shapley and interaction indices. This mathematical facet of calculation makes the Choquet integral model flexible and robust (Grabisch, 1996). To the best of our knowledge, this kind of aggregation has not been previously used for such IR purposes. In this article, we particularly explore the following issues:

1. How to model multidimensional relevance aggregation within dependent criteria. As stated earlier, many pioneering works on multidimensional relevance argue that relevance dimensions usually interact with each other. Likely, we assume, in our context, that relevance dimensions, which are used for aggregation, interact in real IR settings. To do so, we will use the Choquet integral to model interactions between the relevance criteria. One of its main benefits is its ability to represent many kinds of interaction among any set of criteria. This is done thanks to a fuzzy measure $\mu$ (or capacity), defined on each criterion and each subset of criteria $I_{i}$, which enables avoidance of the overestimation (underestimation) caused by possible dependencies between some criteria.

2. How effective is the aggregation proposed within a social search task, namely, the tweet search task? To show the effectiveness of our aggregation approach on real-world IR situations, we propose to instantiate our model on a social (microblogging) IR setting, more particularly, on a tweet search task. We consider jointly three relevant criteria: topicality, recency, and authority, formally described in previous works (Duan et al., 2010; Nagmoti, Teredesai, \& De Cock, 2010). We experimentally show the dependency among these criteria and then show the appropriateness of the Choquet integral in aggregating them.

\section{Background: Aggregation in Decision-Making Problems}

In this section, we present an overview of the aggregation problem in MCDM. Then we introduce a formalization of the MCDM problem and present formal definitions and related notions of aggregation operators.

\section{Aggregation Operators: An Overview}

Aggregation functions involve the ordering of a group of alternatives based on their satisfying a collection of criteria. Research concerning aggregation functions has been conducted in various fields including decision making, knowledge-based systems, and many other areas (James, 2010). The most widely applied aggregation functions are those in the averaging class. The Am and its variations are prominent. Aggregation operators or functions can be roughly classified into several categories: compensatory, noncompensatory, conjunctive, disjunctive, and weighted aggregation approaches (Hwang \& Yoon, 1981). Compensatory operators are based on the assumption that a low score of a given alternative with respect to a high-preference criterion may be compensated for by a high score on another high preference criterion. Compensative operators are included between minimum and maximum; that is, they are neither conjunctive nor disjunctive. The weighted sum is the most representative aggregation function of this class. The global score of each alternative is computed by multiplying the criterion weight by the alternative's performance score obtained on this criterion. Weighted quasi-arithmetic means are also particularly interesting aggregation functions of this family. These functions are used prominently throughout the literature because they generalize a group of common means, for example, the harmonic mean, the quadratic mean, and the power mean, which, in turn, includes as special cases other classical means like the arithmetic and geometric means (Aczel, 1948; Kolmogorov, 1930). Another family extensively studied in the literature is that of Owa functions (Yager, 1988). The fundamental aspect of the Owa operator is the reordering step. More specifically, a given performance score is not associated with a particular weight, but rather a weight is associated with a particular ordered position of the score, which introduces a nonlinearity into the aggregation process.

This provides a means for aggregating scores associated with the satisfaction of multiple criteria, which unifies in one operator the conjunctive and disjunctive behavior. In addition to these families, another form of operators investigated in the context of multicriteria aggregation under uncertainty is the concept of triangular norm (Menger, 1942). The current notion of $t$-norm and its dual operator $t$-conorm were introduced by Schweizer and Sklar (1960, 1983). These operators may be seen as a generalization of the conjunctive "AND" ( $t$-norms) and disjunctive "OR" ( $t$-conorms) logical aggregation functions. Compensatory operators often require the user or the decision maker to specify priorities or preference relations expressed by means of cardinal weights or priority functions over the set of criteria. In contrast, noncompensatory functions, such as the Min or the Max aggregation schemes (Fox \& Shaw, 1994), are generally dominated by just one criterion value, that is, the worst or the best score. The main limitation of these families is the fact that a large number of scores are ignored in the final aggregation process. 
TABLE 3. Particular cases of the Choquet integral.

\begin{tabular}{cc}
\hline & Choquet integral \\
\hline Owa & $\begin{array}{c}\mu_{C}=\sum_{j=0}^{i-1} w_{n-j}, \forall C \text { such that }|C|=i \text {, where }|C| \\
\text { denotes the cardinal of the subset of criteria } C \\
\text { The weight } w_{i} \text { of each criterion } c_{i} \text { is equal to }\left(\mu_{c_{i}}\right) \text { and } \\
\text { for every subset of criteria } C_{1} \in \mathcal{C}, \mu_{C_{1}}=\sum_{c_{i} \in C_{1}} \mu_{c_{i}}\end{array}$ \\
& $\mu_{C_{1}}=\frac{\left|C_{1}\right|}{|\mathcal{C}|}$ \\
\hline
\end{tabular}

Fuzzy integrals such as the Choquet integral and the Sugeno integral (Choquet, 1953) may be considered as a metaclass of aggregation functions. These aggregation operators that are defined with respect to a fuzzy measure are useful for modeling interactions between criteria, such as redundancies among the inputs or complementarity between some criteria. Special cases of the Choquet integral, depending on the fuzzy measure $\mu$, include weighted arithmetic mean (Wam), Owa operator, and Am. In Table 3, we present the corresponding measures to get a particular operator. The Choquet integral has attracted a lot of attention in fuzzy sets, as well as in decision-making communities. However, research into its real-world use in the IR field is still in its infancy.

\section{Multidimensional Relevance Aggregation as an MCDM Problem}

An MCDM method deals with the process of making decisions in the presence of multiple objectives or alternatives (Triantaphyllou, 2000). The main goal of MCDM methods is to assist a decision maker in selecting the best alternative(s) from a number of given ones $\mathcal{A}=\left\{a_{1}, a_{2}, \ldots, a_{M}\right\}$ under the presence of multiple criteria $\mathcal{C}=\left\{c_{1}, c_{2}, \ldots, c_{N}\right\}$ and diverse criterion preferences.

The point of departure for any MCDM technique is the generation of the discrete set of alternatives, the formulation of the set of criteria, and then the evaluation of the impact of each alternative on every criterion (Jankowski, 1995). The estimated impacts of alternatives $a_{j}(1 \leq j \leq M)$ on every criterion $c_{i}(1 \leq j \leq N)$ are called performance scores (or evaluations), which we denote $C_{i j}$, defined with respect to a partial preference order $\preceq_{c_{i}}$. Thereafter, preferences on the set of criteria may be formulated as is the case for the weighted averaging operator, in a cardinal vector of normalized criterion preference weights $W=\left(w_{l}, w_{2}, \ldots, w_{n}\right)$ (with $0 \leq w_{i} \leq 1$ and $n$ is then number of criteria). In the final step, performance scores are aggregated into a single one, for each alternative $a_{j}(1 \leq j \leq M)$, using an appropriate aggregation function $\mathcal{F}\left(C_{1 j}, C_{2 j}, \ldots, C_{N j}\right)$. The result is then an ordered set of alternatives with respect to the defined preferences.

Based on the multidimensional property of relevance, detailed in the Multidimensional Relevance Aggregation in IR section, we suggest to model the multidimensional relevance aggregation as an MCDM problem. Thus, the set of alternatives is represented by the document collection, and criteria are the possible relevance dimensions. Our research considers the following retrieval setting: A user $U$ interacts with a document space $\mathcal{D}=\left\{d_{1}, d_{2}, \ldots, d_{M}\right\}$ with a typical search engine through an information need stated by means of query $q_{k}$. In this setting, the user's relevance judgment is affected by a set of criteria $\mathcal{C}=\left\{c_{1}, c_{2}, \ldots, c_{N}\right\}$, each of which has a given importance degree or preference. The aggregation problem consists of combining the performance scores $C_{i j}$ of each document with respect to all the relevance criteria. As we deal with an IR setting, we denote $C_{i j}$ by $R S V_{c_{i}}\left(q_{k}, d_{j}\right)$ (i.e., retrieval status value), obtained for each document $d_{j}$, on each single criterion $c_{j}$ in response to a given query $q_{k}$. Then the result consists of the global score denoted by $R S V_{\left\{c_{1}, \ldots, c_{N}\right\}}\left(q_{k}, d_{j}\right)$, with respect to a global preference relation $\preceq_{c}$ on the set of all criteria. More formally, an aggregation operator is expressed as follows:

$$
\mathcal{F}:\left\{\begin{array}{l}
\mathbb{R}^{N} \rightarrow \mathbb{R} \\
\left(R S V_{c_{1}}\left(q_{k}, d_{j}\right) \times \ldots \times R S V_{c_{N}}\left(q_{k}, d_{j}\right)\right) \\
\rightarrow \mathcal{F}\left(R S V_{c_{1}}\left(q_{k}, d_{j}\right), \ldots, R S V_{c_{N}}\left(q_{k}, d_{j}\right)\right)
\end{array}\right.
$$

$R S V_{c_{i}}\left(q_{k}, d_{j}\right)$ may also be interpreted as the satisfaction degree of document $d_{j}$ with respect to criterion $c_{i}$. To avoid overestimation (underestimation) of the global relevance scores by those having high (low) values with respect to some criteria, we normalized the performance scores before aggregation by scaling them into the range $[0 \ldots 1]$. The aggregation function that is used in our approach is the discrete Choquet integral (Choquet, 1953; Grabisch, 1996). This function allows us to define a weight not only on each criterion, but also on each subset of criteria, which gives rise to a more flexible representation of interaction among criteria (Grabisch, 1996).

\section{iAggregator: A Multidimensional Relevance Aggregation Operator Using the Choquet Integral}

In the remainder of this article, we rely on the Choquet integral fuzzy-based function to solve the multidimensional relevance aggregation problem. The choice of this operator is mainly motivated by its flexible representation of complex interactions among criteria, especially in situations involving redundant or complementary information. A first step that should be performed before proceeding to the multicriteria aggregation with the Choquet integral is the definition of the fuzzy measure values or capacities $\mu_{\left\{c_{i}\right\}}$ on each criterion and each subset of relevance criteria.

\section{Definition of the Fuzzy Measure on the Set of Relevance Dimensions}

Let $\mathcal{C}$ be the set of criteria (i.e., the relevance dimensions) and $I_{\mathcal{C}}$ be the set of all possible subsets of criteria from $\mathcal{C}$. A fuzzy measure is a function $\mu$ from $I_{\mathcal{C}}$ to $[0 \ldots 1]$ such that 


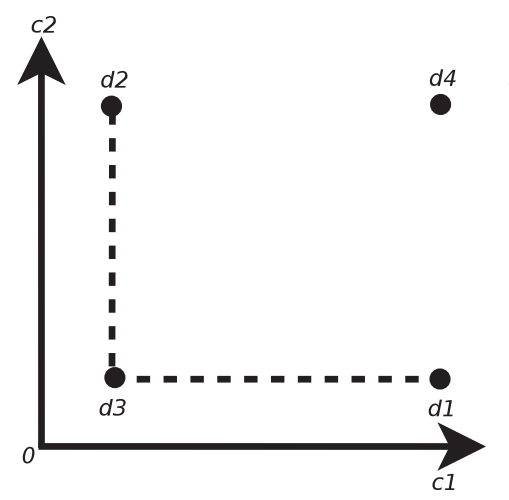

(a) A postive interaction

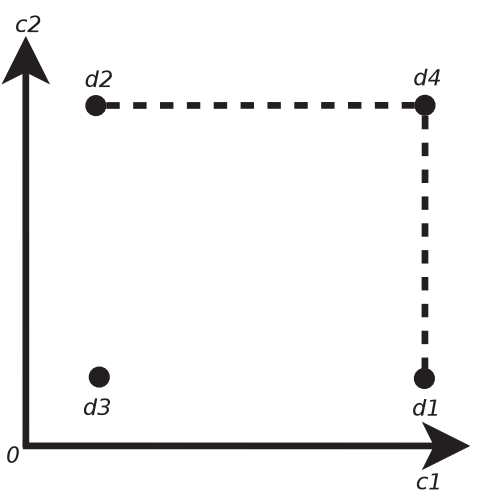

(b) A negative interaction

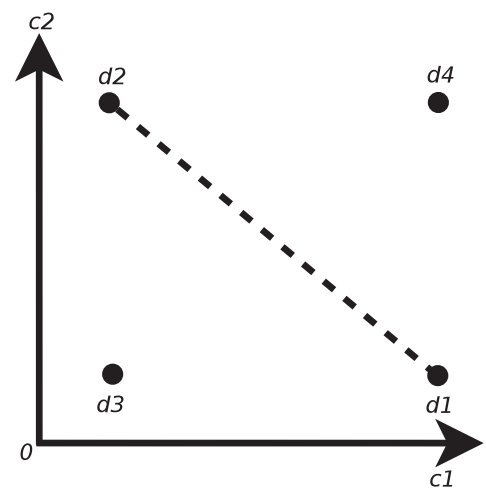

(c) An independent interaction

FIG. 1. Possible interactions between the set of criteria.

$\forall I_{C_{1}}, \quad I_{C_{2}} \in I_{\mathcal{C}}$, if $\left(I_{C_{1}} \subseteq I_{C_{2}}\right)$, then $\mu\left(I_{C_{1}}\right) \leq \mu\left(I_{C_{2}}\right)$, with $\mu\left(I_{\varnothing}\right)=0$ and $\mu\left(I_{\mathcal{C}}\right)=1 . \mu\left(I_{C_{i}}\right)$ can be interpreted as the importance degree of the combination of the subset of criteria $I_{C_{i}}$, or similarly, its power to make decisions alone without the remaining relevance criteria. For the sake of notational simplicity, $\mu\left(I_{C_{i}}\right)$ will be denoted in the remainder by $\mu_{C_{i}}$.

Assume now that we have a document collection $\mathcal{D}$ and $d_{j} \in \mathcal{D}$. The global document's score of $d_{j}$ given by the Choquet integral with respect to the fuzzy measure $\mu$ with respect to a set of $N$ relevance criteria $\mathcal{C}$ is defined by:

$$
C h_{\mu}\left(C_{1 j}, \ldots, C_{N j}\right)=\sum_{i=1, \ldots, N}\left(c_{(i) j}-c_{(i-1) j}\right) \cdot \mu_{C_{(i)}}
$$

where $c_{(i) j}$ is the score obtained on a given criterion. The notation $c_{(\cdot) j}$ indicates that the indices have been permuted such that $0 \leq c_{(1) j} \cdots \leq c_{(N) j} . C_{(i)}=\left\{c_{i}, \ldots, c_{N}\right\}$ is the set of relevance criteria with $C_{(0)}=0$ and $\mu_{C_{(1)}}=1$, and $C_{i j}$ is the performance score ${ }^{2}$ of $d_{j}$ with respect to criterion $c_{i}$.

Obviously, the crucial part of using the Choquet integral is the modeling of interactions between criteria via the fuzzy measure $\mu$. Because the latter can model correlations or dependencies among criteria, which are relevance dimensions in our case, it is worth mentioning that there are three possible kinds of interactions represented in Figure 1. The $x$-axis and $y$-axis represent the performance scores of the four documents $d_{1}, d_{2}, d_{3}$, and $d_{4}$ with respect to the criteria $c_{1}$ and $c_{2}$, respectively. The documents connected by dashed lines have the same importance degree.

- Positive interaction: Can also be called complementarity; when the global weight of two relevance criteria is greater than their individual weights: $\mu_{c_{i}, c_{j}}>\mu_{c_{i}}+\mu_{c_{j}}$. This inequality can also be expressed as follows: The contribution of criterion $c_{j}$ to every combination of criteria that contains $c_{i}$ is strictly greater than that of $c_{j}$ to the same combination when $c_{i}$ is

${ }^{2}$ The difference between $c_{(i) j}$ and $C_{i j}$ is that the performance scores $c_{(i) j}$ have been permuted before computing the overall scores. excluded. In this case, criteria $c_{i}$ and $c_{j}$ are said to be negatively correlated. In other words, we say that the satisfaction of only one single relevance criterion should produce a very weak effect compared with the satisfaction of both criteria. Intuitively, in an IR setting, this kind of preference favors documents that are satisfied equivalently by all sets of criteria, rather than those that are overestimated by one single relevance criterion. For instance, in Figure 1a, document $d_{4}$ should be preferred to documents $d_{2}$ and $d_{3}$ because they do not satisfy equivalently the two criteria $c_{1}$ and $c_{2}$.

- Negative interaction: When the global weight of two relevance criteria is smaller than their individual weights: $\mu_{c_{i}, c_{j}}<\mu_{c_{i}}+\mu_{c_{j}}$. We say that the union of criteria does not bring anything and the criteria are considered to act disjunctively. Thus, they are said to be redundant. This is indeed a key point about the Choquet integral, because it smooths the bias effect of redundant relevance criteria in the global documents evaluation. This is done by associating a small importance degree $\mu_{c_{i}, c_{j}}$ to the subset of the two redundant relevance criteria, compared with their single importance weights $\mu_{c_{i}}$ and $\mu_{c_{j}}$. From Figure $1 \mathrm{~b}$ we remark that document $d_{4}$ has the same importance as documents $d_{2}$ and $d_{3}$, because the satisfaction of one criterion from $c_{1}$ or $c_{2}$, which are in turn redundant, is sufficient to judge a document as a relevant one.

- Independence: When there is no correlation between the set of criteria, the fuzzy measure is said to be additive: $\mu_{c_{i}, c_{j}}=\mu_{c_{i}}+\mu_{c_{j}}$. The Wam is an example of such functions that allow this independence between criteria. Accordingly, the importance of the inputs is taken into account and the weight of each criterion indicates its importance.

To facilitate the task of interpreting the behavior of the Choquet integral and the interaction phenomena between the relevance criteria, we introduce the Importance index (or Shapley value) (Shapley, 1953) and the Interaction index modeled by the underlying fuzzy measure.

Definition 1. Importance index: Let $\mu_{c_{i}}$ be the weight of relevance criterion $c_{i}$ and $\mu_{C r \cup c_{i}}$ its marginal contribution to each subset $C r \in \mathcal{C}$ of other criteria. The importance index (Shapley, 1953) of $c_{i}$ with respect to a fuzzy measure $\mu$ is then defined as the mean of all these contributions: 


$$
\phi_{\mu}\left(c_{i}\right)=\sum_{C r \subseteq \mathcal{C} \backslash\left(c_{i}\right)} \frac{(N-|C r|-1) ! .|C r| !}{N !}\left[\mu_{C r} \cdot \mu_{\left(C r \cup c_{i}\right)}\right]
$$

$\phi_{\mu}\left(c_{i}\right)$ measures the average contribution that criterion $\left(c_{i}\right)$ brings to all the possible combinations of criteria.

This Importance index gives no information on the phenomena of interaction existing among the relevance criteria. The overall importance of criterion $c_{i}$ is not solely determined by its weight $\mu_{c_{i}}$ but also by its contribution to each subset of other criteria. Then, to quantify the degree of interaction between a subset of criteria, we introduce the concept of Interaction index (Murofushi \& Soneda, 1993).

Definition 2. Interaction index: Let $\left(\Delta_{c_{i} c_{j}} \mu_{C r}\right)$, with $C r=\mathcal{C} \backslash\left\{c_{i}, c_{j}\right\}$, be the difference between the marginal contribution of criterion $c_{j}$ to every combination of criteria that contains criterion $c_{i}$, and a combination from which criterion $c_{i}$ is excluded:

$$
\left(\Delta_{c_{i} c_{j}} \mu_{C r}\right)=\left[\mu_{\left(\left\{c_{i} c_{j}\right] \cup C r\right)}-\mu_{\left(c_{i} \cup C r\right)}\right]-\left[\mu_{\left(c_{i} \cup C r\right)}-\mu_{C r}\right]
$$

This expression is defined to appraise the strength among two criteria $c_{i}$ and $c_{j}$. When this latter expression is positive (negative) for any $C r \in \mathcal{C} \backslash\left\{c_{i}, c_{j}\right\}$, we say that both criteria $c_{i}$ and $c_{j}$ positively (negatively) interact (i.e., the contribution of criterion $c_{j}$ is higher with the presence of criterion $c_{i}$ ). The interaction index among two measures is thus defined as follows:

$$
I_{\mu}\left(c_{i}, c_{j}\right)=\sum_{C r \subseteq \mathcal{C} \backslash\left\{c_{i}, c_{j}\right\}} \frac{(N-|C r|-2) ! .|C r| !}{(N-1) !}\left(\Delta_{c_{i} c_{j}} \mu_{C r}\right)
$$

The interaction value, which falls into the interval $[-1 \ldots 1]$, is zero when both criteria are independent and is positive (negative) whenever the interaction between them is positive (negative).

\section{Design of a Multidimensional Relevance Function}

The overall relevance score of document $d_{j}$, given by the Choquet integral with respect to a fuzzy measure $\mu$ and according to the set $\mathcal{C}$ of $N$ relevance criteria, is defined by:

$$
\begin{aligned}
& R S V_{\left(c_{1}, c_{2}, \ldots, c_{N}\right)}\left(q_{k}, d_{j}\right) \\
& \quad=C h_{\mu}\left(R S V_{c_{1}}\left(q_{k}, d_{j}\right), \ldots, R S V_{c_{N}}\left(q_{k}, d_{j}\right)\right) \\
& \quad=\sum_{i=1}^{N}\left(r s v_{(i) j}-r s v_{(i-1) j}\right) \cdot \mu_{C_{(i)}}
\end{aligned}
$$

where $C h_{\mu}$ is the Choquet aggregation function, $r s v_{(i) j}$ is the permutation of $R S V\left(q_{k}, d_{j}\right)$ on criterion $c_{i}$ such that $\left(0 \leq r s v_{(1) j} \leq \ldots \leq r s v_{(N) j}\right)$, and $C_{(i)}=\left\{c_{i}, \ldots, c_{N}\right\}$ is a set of relevance criteria with $\mu_{C_{(0)}}=0$ and $\mu_{C_{(1)}}=1$.

Once the Choquet operator and the interactions between criteria are defined, we present the mechanism used for the identification of the fuzzy measures. In fact, the proposed methods in the literature for capacity identification differ according to the preferential information they require as input. Most of them are classified as optimization problems
(Grabisch et al., 2008). In this article, we rely on the leastsquares-based approach for the identification of capacities representing preferences on the relevance dimensions. This method is the most extensively used approach in the literature (Grabisch, 2002). First, we suppose to have initially a small selected subset of documents $\mathcal{D}$ that can be seen as a learning set. A ground truth is built with respect to a set of relevance criteria. Suppose now that we know the performance scores $R S V_{c_{i}}\left(q, d_{j}\right)$ assigned to each document $d_{j}$ (with respect to $c_{i}$ ) from the chosen subset of documents. In addition, we also suppose that we know the desired overall relevance scores $R S V_{\left\{c_{1}, \ldots, c_{N}\right\}}^{*}\left(q, d_{j}\right)$ for each document. The initial preferences can be formalized as follows:

- Given the partial order relation $\preccurlyeq_{c_{i}}$ (ranking of documents with respect to criterion $c_{i}$ ), the relation $d_{1} \preccurlyeq_{c_{i}} d_{2}$ can be interpreted as $d_{1}$ is more relevant than $d_{2}$ according to the relevance criteria $c_{i}$. In the context of the Choquet integral, this relation is translated as $C_{\mu}\left(R S V_{c_{i}}\left(q, d_{1}\right)\right) \leq$ $C h_{\mu}\left(R S V_{c_{i}}\left(q, d_{2}\right)\right)$

- $C_{\mu}\left(R S V_{c_{i}}\left(q, d_{1}\right)\right) \simeq\left(R S V_{c_{i}}\left(q, d_{2}\right)\right)$ can be interpreted as the degree of satisfaction of $d_{2}$ with respect to the relevance criterion $c_{i}$ is the same as that of $d_{1}\left(d_{1} \simeq_{c_{i}} d_{2}\right)$.

- A partial preference order on the set of criteria $\preccurlyeq_{\mathcal{C}}$, that is, $c_{1} \preccurlyeq_{\mathcal{C}} c_{2}$ is interpreted as $c_{1}$ is more important than $c_{2}$.

- A partial preference order on the subset of criteria $\preccurlyeq_{I}$, that is, $I_{C_{1}} \preccurlyeq{ }_{I} I_{C_{2}}$ is interpreted as the combination of criteria $I_{C_{2}}$ is more important than the combination of the subset of criteria $I_{C_{1}}$.

Suppose now that we know the performance scores $R S V_{c_{i}}\left(q, d_{j}\right)$ that should be assigned to each document $d_{j} \in \mathcal{D}$ (with respect to criterion $c_{i}$ ). Then the main objective of the least-squares-based approach is to minimize the total squared error $E^{2}$ between the desired global relevance score, given on each document $d_{j}$, and the global scores calculated by the Choquet integral as follows:

$$
\begin{aligned}
E^{2}= & \sum_{k=1}^{l}\left(C h_{\mu}\left(R S V_{c_{1}}\left(q, d_{j}\right), \ldots, R S V_{c_{N}}\left(q, d_{j}\right)\right)\right. \\
& \left.-R S V_{\left\{c_{1}, \ldots, c_{N}\right\}}^{*}\left(q, d_{j}\right)\right)^{2}
\end{aligned}
$$

This optimization process is discussed in detail in the Tuning the Choquet Capacities section.

\section{Experimental Evaluation Setting}

The proposed multidimensional relevance operator is evaluated within a social IR setting, namely, tweet search task. In this section, we present the experimental evaluation setup, the data set used, as well as the evaluation protocol.

\section{Tweet Search Task}

Seeking for information over microblogging spaces becomes a challenging task because of the increasing amount of published information. One of the most 
well-known microblogging networking services that enables users to broadcast informations is Twitter. ${ }^{3}$ The TREC 2011 Microblog Track (Ounis et al., 2011) defines tweet search as a real-time ad hoc task where the users are interested in the most recent and relevant information. Recent works addressing the tweet search integrate a number of interesting features that were identified with potential implications in the final ranking of documents (Duan et al., 2010; Nagmoti et al., 2010). A number of proposed criteria include, for instance, textual features, user's preferences, microblogging, and social network features. In this work, we evaluate our Choquet integral-based operator in a tweet search setting considering three relevance criteria: topicality, recency, and authority. The aggregation of these criteria with the Choquet integral with respect to a fuzzy measure $\mu$, in response to a user' query $q$, is defined as:

$$
\begin{gathered}
C h_{\mu}\left(R S V_{T o}\left(q, T_{j}\right), R S V_{A u}\left(q, T_{j}\right), R S V_{R e}\left(q, T_{j}\right)\right) \\
=\sum_{i=1}^{3}\left(r s v_{(i) j}-r s v_{(i-1) j}\right) \cdot \mu_{C_{(i)}}
\end{gathered}
$$

where $T_{j}$ is a tweet (or microblog), $r s v_{(i) j}$ indicates that the performance score ${ }^{4}$ considering criterion $c_{i}$ on query $q$ has been permuted such that $0 \leq r s v_{(1) j} \leq r s v_{(2) j} \leq r s v_{(3) j}$ (i.e., $r s v_{(i) j}$ is the $i$-th smallest $d_{j}$ score obtained on criterion $\left.c_{i} \in\{T o, A u, R e\}\right)$. Note that $C_{(i)}=\left\{c_{i}, \ldots, c_{3}\right\}$, and $C h_{\mu}$ is the global score that defines the final ranking of each tweet with respect to the three criteria.

In the following list, we present a formal description of these relevance criteria in our evaluation setting.

- Topicality: a content relevance criterion that describes the relevance between queries and tweets. To deal with this criterion, we propose to use the Okapi BM25 ranking function to rank tweets according to their relevance to a given search query. The standard BM25 weighting function is defined as follows:

$$
B M 25(T, Q)=\sum_{q_{i} \in Q} \frac{\operatorname{Idf}\left(q_{i}\right) \cdot \operatorname{tf}\left(q_{i}, T\right) \cdot\left(k_{1}+1\right)}{\operatorname{tf}\left(q_{i}, T\right)+k_{1}\left(1-b+b \frac{\text { Length }(T)}{a v g_{\text {length }}}\right)}
$$

where $\operatorname{Idf}\left(q_{i}\right)$ is the inverse document frequency, Length(T) denotes the length of tweet $T$, and $a v g_{\text {length }}$ represents the average length of tweets in the collection.

- Authority: represents the influence of tweets' authors in Twitter. We define it as it was presented by Nagmoti et al. (2010): $A u(T)=A u_{n b}(T)+A u_{m e}(T)$, where:

- $A u_{n b}(T)$ is the total number of tweets, to favor tweets published by influential users. $A u_{n b}(T)=N\left(a_{i}(T)\right)$, where $a_{i}(T)$ represents the author of tweet $T$ and $N\left(a_{i}(T)\right)$ denotes the number of tweets published by $a_{i}$.

- $A u_{m e}(T)$ is number of mentions, that is, the more an author has been cited (or mentioned), the more popular he is. It is

\footnotetext{
${ }^{3}$ http://www.twitter.com

${ }^{4}$ All the performance scores are normalized so that they belong to $[0 \ldots 1]$.
}

TABLE 4. Statistics of the TREC 2011 and 2012 Microblog tracks data set.

\begin{tabular}{lr}
\hline Tweets & $16,141,812$ \\
Null tweets & $1,204,053$ \\
Unique terms & $7,781,775$ \\
Microbloggers & $5,356,432$ \\
TREC Microblog 2011 Topics & 49 \\
TREC Microblog 2012 Topics & 60 \\
\hline
\end{tabular}

defined as $A u_{m e}(T)=N_{m e}\left(a_{i}(T)\right)$, whereas $N_{m e}\left(a_{i}(T)\right)$ denotes the number of times the author of tweet $T$ has been mentioned in the collection.

- Recency: the difference between the time a tweet was published $T p(T)$ and the query submission's time stamp $T s(Q)$. $\operatorname{Re}(T)=T_{s}(Q)-T_{p}(T)$. Because we are interested in attempting the real-time ad hoc search task, all the tweets that occurred after the query time are excluded from the scoring.

\section{Experimental Data Sets}

We exploit the data sets distributed by TREC 2011 and 2012 Microblog tracks (Ounis et al., 2011, 2012). The Microblog Track is a focus area within TREC to examine search issues in Twitter. The Tweets 2011 corpus includes approximately 16 million tweets published over 16 days. The real-time ad hoc task of the TREC Microblog 2011 track includes 49 time-stamped topics that serve as queries. Each topic represents an information need at a specific point in time. Actually, we exploit the 49 topics of the TREC Microblog 2011 track for the capacities learning, and we used the 60 TREC Microblog 2012 track for testing (Ounis et al., 2012). The general data set statistics are reported in Table 4.

We use the Terrier ${ }^{5}$ search engine for indexing and retrieval. Because the task focuses on English tweets only, we eliminated the non-English tweets using a simple language identifier tool. We also used some regular expressions to filter out some common types of tokens known in Twitter, but we did not filter the terms starting with the @ or \# symbols. Although spam tweets are included, we did not perform any further processing because the main concern of our work is the multicriteria relevance assessment.

\section{Evaluation Protocol}

We adopt an evaluation protocol, consisting of two steps, as described in the following list.

- Training step: This step consists of learning the Choquet capacities that are of use within each relevance dimension and each subset of relevance criteria in the aggregation process. Thus, we propose to exploit the TREC Microblog 2011 track (49) topics to test different combinations of capacities. Because the relevance assessments relative to the track are

\footnotetext{
${ }^{5}$ http://terrier.org
} 
TABLE 5. Rank correlation analysis of the relevance criteria in the tweet search task.

\begin{tabular}{|c|c|c|c|c|c|c|c|}
\hline \multicolumn{4}{|c|}{ Rank correlation coefficient for the single criteria rankings } & \multicolumn{4}{|c|}{ Rank correlation coefficient for the subset of criteria } \\
\hline Criterion & Topicality $(T)$ & Recency $(R)$ & Authority $(A)$ & Criterion & $\{T, R\}$ & $\{T, A\}$ & $\{R, A\}$ \\
\hline Topicality & 1 & .1580 & .0013 & $\{T, R\}$ & 1 & .2290 & .1210 \\
\hline Recency & - & 1 & .0010 & $\{T, A\}$ & - & 1 & -.1030 \\
\hline Authority & - & - & 1 & $\{R, A\}$ & - & - & 1 \\
\hline
\end{tabular}

available, we select the best capacities that optimize our aggregation model effectiveness in such an IR task.

- Testing step: This step consists of testing the iAggregator effectiveness based on the TREC Microblog 2012 track (60) topics. To assess the effectiveness of our approach, we rely on the precisions at rank 10, 20, 30 denoted, respectively, by P@10, P@20, P@30, and mean average precision (MAP).We note that P@30 is used officially to evaluate the retrieval performances of the participating groups in the Microblog Tracks. These evaluation measures are computed with the standard trec eval ${ }^{6}$ tool.

Moreover, as is case for learning-to-rank methods (Liu, 2009; Macdonald, Santos, \& Ounis, 2013), our Choquetbased approach involves the use of a sample of top-ranked documents returned in response to a given query, initially based on the BM25 standard weighting model. Then these documents are reranked with respect to the other criteria and the aggregation is done on the three relevance dimensions. This manner in which the approach is deployed is also used by most of the TREC Microblog participants (Liang, Qiang, Hong, Fei, \& Yang, 2012; Miyanishi, Seki, \& Uehara, 2012), who used instead of BM25 a language model ranking. The participants are required to return top-ranked tweets before a query time per document according to their relevance score.

\section{Results and Discussion}

In this section, we evaluate the effectiveness of iAggregator. We start first by introducing the evaluation objectives, as well as the method used to tune the Choquet capacities, and then discuss the retrieval results.

\section{Evaluation Objectives}

The aim of the experiments presented in the remainder is twofold:

- Evaluate the impact of criteria interactions. We show the ability of the Choquet integral in combining the relevance of dependent dimensions. The dependency property is estimated using a ranking correlation analysis. We also exploit the interaction and importance indices given through the fuzzy measure (cf. Definition of the Fuzzy Measure on the Set of Relevance Dimensions section) to estimate the interactions

\footnotetext{
${ }^{6} \mathrm{http}: / /$ trec.nist.gov/trec_eval
}

between the considered criteria. The impact of the criteria dependency on the retrieval performances is also discussed.

- Compare iAggregator with state-of-the-art aggregation operators. We compare our approach versus the Am, the Wam, and the linear combination mechanism, as well as the Min, Max, Owa (Yager, 1988), OWmin (Dubois \& Prade, 1996), And, and Scoring aggregation operators (Costa Pereira et al., 2009). Afterward, we evaluate iAggregator with three conventional state-of-the-art learning-to-rank algorithms, namely, RankNet (Burges et al., 2005), RankSVM (Joachims, 2006), and ListNet (Cao, Qin, Liu, Tsai, \& Li, 2007).

\section{Correlation Analysis of the Relevance Dimensions}

One of the main advantages in using the Choquet integral is its ability to aggregate interacting or correlated criteria. We present a correlation analysis of the relevance dimensions through the Kendall's tau $(\tau)$ coefficient (Kendall, 1938). Our objective is to show the interaction that could exist among the set of the considered criteria and to justify the use of the Choquet integral in such problems, considering the wide range of works proving this fact (Carterette et al., 2011; Wei et al., 2010).

Kendall's $\tau$ correlation coefficient analyzes the agreement between two rankings considering concordant and discordant pairs. We analyze the agreement between tweet rankings returned by each considered criterion solely on one side and subsets of criteria on the other side. The more similar (reversed) the rankings are, the closer to 1 the correlation coefficient $\tau$ is $(-1)$. If the rankings are independent, then we would expect the coefficient to be approximately equal to zero. Table 5 shows the rank correlation coefficient for the individual criteria rankings and for the subset of relevance criteria rankings. Each coefficient is computed over the TREC Microblog 2012 track topics rankings. The global results are averaged over the resulted documents from each ranking. At a glance, Table 5 highlights that recency and topicality are significantly correlated, whereas authority seems to be independent and less important. From Table 5 we notice, unlikely, that authority impacts ranking in the presence of both topicality and recency. One can see that the impact is more important in presence of topicality, which is quite expected.

To present an in-depth understanding of this interaction phenomena, we show in the following the Shapley values, as well as the interaction indices, obtained through the fuzzy measure within the TREC Microblog 2011 data set. These 
TABLE 6. Criteria importance and interaction indices.

\begin{tabular}{lccc}
\hline Criterion & Topicality & Recency & Authority \\
\hline $\begin{array}{l}\text { Criterion importance index } \\
\text { Criteria interaction index }\end{array}$ & $\mathbf{0 . 6 3}$ & $\mathbf{0 . 2 5}$ & $\mathbf{0 . 1 2}$ \\
Topicality & - & $+\mathbf{0 . 1 8}$ & +0.01 \\
Recency & - & - & -0.10 \\
Authority & - & - & - \\
\hline
\end{tabular}

parameters provide meaningful information that can be used to interpret the resulting model behavior.

As shown in Table 6, given the marginal contribution of the content matching criterion in this IR task, we notice the high importance index of topicality with a value of 0.631 . The recency relevance criterion is also given quite high importance compared with the authority relevance dimension. This is not surprising given that we deal with a realtime ad hoc task and we are interested in the most relevant and recent tweets (Ounis et al., 2011). To analyze the Interaction phenomena existing among these relevance criteria and quantify its degree, we report in Table 6 the values of the Interaction index between the three relevance criteria: topicality, recency, and authority. From Table 6 we can also remark that the authority criterion is not important and it does not bring any contribution when it is combined with topical relevance criteria.

Also notice a positive interaction between the topicality and recency relevance criteria. This explains the higher contribution of these two criteria on the overall global scoring when they are present together, and this concords with the aim of the considered IR setting. These results are in concordance with those obtained by Kendall's $\tau$ correlation coefficient, which prove the dependencies between the relevance criteria and motivate the use of the Choquet integral to aggregate them.

\section{Tuning the Choquet Capacities}

In this section, we study the tuning of the capacity values that should be assigned to each criterion and each subset of criteria before computing the global Choquet scores. Because we have the relevance assessments corresponding to the TREC Microblog 2011 track topics, we used the least-squares-based approach (cf. Design of a Multidimensional Relevance Function section) to tune the best combination of capacities that should be attributed to the relevance dimensions. Actually, each combination $\mu^{(i)}$ is composed by the following subsets of criteria:

$$
\begin{aligned}
\mu^{(i)}= & \left\{\mu_{\{t o p i c a l i t y\}}, \mu_{\{\text {authority }\}}, \mu_{\{\text {recency }\}}, \mu_{\{t o p i c a l i t y, \text { authority }\}},\right. \\
& \left.\mu_{\{\text {topicality, recency }\}}, \mu_{\{\text {recency, authority\} }}\right\} .
\end{aligned}
$$

The different experimental capacity combinations $\mu_{\{.\}}$used within each criterion and each combination of criteria fall into $[0 \ldots 1]$ and are computed with a step equal to .1. The method used for assigning capacity values for these relevance criteria is described as follows:
TABLE 7. Notation used within Algorithm 1.

\begin{tabular}{ll}
\hline Notation & \multicolumn{1}{c}{ Description } \\
\hline$Q_{\text {learn }}$ & The set of queries used to train the capacity values \\
$\mathcal{D}$ & The document collection \\
qrels & The set of user's relevance assessments including relevant \\
& documents for each query $q \in Q_{\text {learn }}$ \\
& qrels $(q)$ : relevant documents of query $q$ \\
& The set of the experimented capacity combination values; \\
$\mathcal{S}_{\mu^{(l)}}$ & each combination $\mu^{(i)} \in \mathcal{S}_{\mu^{(l)}}$ contains the capacities \\
& values of all the set and subsets of criteria; for instance, \\
& in the case of three criteria, each $\mu^{(i)}$ involves \\
& $\left(\left\{\mu_{c_{1}} ; \mu_{c_{2}} ; \mu_{c_{3}} ; \mu_{c_{1}, c_{2}} ; \mu_{c_{1}, c_{3}} ; \mu_{c_{2}, c_{3}}\right\}\right)$ \\
\hline
\end{tabular}

- Step 1: We start by assigning higher capacity values to the topical criterion, and we start by .8. The capacity values of the recency and authority criteria are, respectively, equal to .1 and .1 , that is, the sum of the three relevance criteria capacities is 1. The capacity values of each subset of criteria are the sum of its single capacity criteria. Then, we decrement the topical capacity value by .1 and we increment the recency capacity value, with the same step. This process is repeated until the topical capacity reaches .1 and the recency criterion capacity reaches .8 .

- Step 2: We assign the recency criterion a high capacity, equal to .8. We decrement the recency capacity and we increment the authority criterion capacity until it reaches .8 (the step is .1).

- Step 3: We assign the authority criterion a high capacity, equal to .8. We decrement the authority capacity and we increment the topicality criterion capacity until it reaches .8 (the step is .1).

The method is detailed in Algorithm 1, while Table 7 describes the notations used within Algorithm 1.

Algorithm 1 Identification of the Fuzzy Measures

Data: The set of queries $Q_{\text {learn }}$, document collection, the set qrels of relevance assessments, capacity combinations.

Result: Capacity values $\mu_{\{i\}}$ of all the criteria and the subset of criteria.

1. For each query $q_{k} \in Q_{\text {learn }}$ do

2. For each capacity combination value do

3. Compute the $P @ X$ of the returned documents in response to query $q_{k}$.

4. End for

5. End for

6. Select the combination of capacities $\mu^{(*)}$ that gives the best average $P @ X$ on the training set $Q_{\text {learn }}$.

7. Select a subset of returned relevant documents $d_{j} \in R\left(q_{k}\right)$ such as $R\left(q_{k}\right) \subset q$ rels $\left(q_{k}\right)$ with their given partial and global scores based on combination $\mu^{(*)}$.

8. Select a subset of returned nonrelevant documents $d_{n r} \in N R\left(q_{k}\right)$ such as and $d_{n r} \notin q r e l s\left(q_{k}\right)$ with their given partial and global scores based on combination $\mu^{(*)}$.

9. Assign to each document $d_{j} \in R\left(q_{k}\right)$ higher (partial and global) scores than each document $d_{n r} \in N R\left(q_{k}\right)$ (even if they are ranked on the bottom).

10. Apply the least-squares-based approach on the set of assigned scores, return the outcome $\mu^{(* *)}$. 


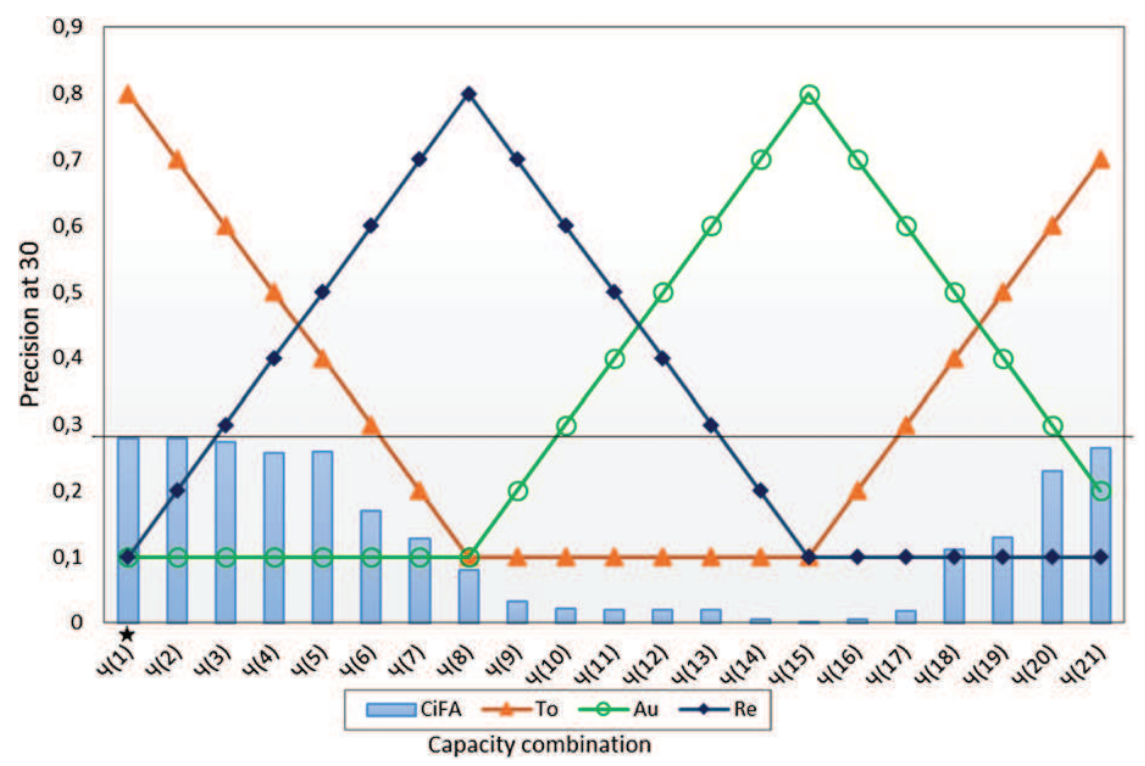

FIG. 2. iAggregator effectiveness within different capacity combination values on the learning phase. [Color figure can be viewed in the online issue, which is available at wileyonlinelibrary.com.]

We denote by $\left(\mu^{(1)}\right)$ the best combination obtained during the learning phase, which gives the higher average value of P@30 on the set of the TREC Microblog 2011 learning topics. This combination includes the following values: $\mu_{T}=.8, \mu_{A}=.1, \mu_{R}=.1, \mu_{T, A}=.9, \mu_{T, R}=.9, \mu_{A, R}=.2$, where $T, A$, and $R$ represent, respectively, topicality, authority, and recency.

Figure 2 plots the performance of our approach within the TREC Microblog 2011 track topics, using the test combinations of capacities, which are obtained as described earlier. The $x$-axis represents the 21 trained capacities combinations $\mu^{(i)} \in \mathcal{S}_{\mu^{(l)}}$, which correspond to the fuzzy measures values of each criterion and each subset of criteria, as previously illustrated. The $y$-axis represents the results obtained in terms of P@30 after application of the Choquet integral within the aforementioned relevance criteria (To, $A u, R e)$. The highlighted value in Figure $2\left(\mu^{(1)}\right)$ indicates the best combination obtained during the learning phase because it gives the higher average value of $\mathrm{P} @ 30$ on the set of the TREC Microblog 2011 learning topics $\left(Q_{\text {learn }}\right)$.

As may be seen from the returned capacity combination values of $\left(\mu^{(1)}\right)$ and from the other combination values in Figure 2, iAggregator is likely to be penalized for showing any preference for tweets, for which the topical and authority criteria are important. In fact, iAggregator is underperformed for topics for which tweets' scores are important with respect to criterion authority; those tweets occur deeper in the ranking. Nevertheless, more recent topically scored tweets are more likely to be relevant, and this explains the positive interaction for both criteria. Therefore, because the system performs well when the topical and recency criteria are important, we consider it a "success" at dealing with the real-time TREC Microblog task.
Furthermore, the capacity combination returned by the least-squares-based approach $\mu^{(*)}$ includes the following criteria capacity values: $\left(\mu_{T o}=0.633, \quad \mu_{R e}=0.204\right.$, $\left.\mu_{A u}=0.153, \mu_{(T o, R e)}=0.961, \mu_{(T o, A u)}=-0.210, \mu_{(R e, A u)}=-0.5\right)$. Our approach gives more importance to the topical and recency criteria. This fits well the Microblog track aim, because users are generally interested in tweets arriving at a specific time and concerning something happening now. We notice that the capacity values on the subsets $\{T o, A u\}$ and $\{R e, A u\}$ are negative. Thus, the contribution of the topicality relevance criterion to every combination of criteria that does not contain authority is greater than its contribution when the criterion authority is highly scored. The same fact holds for the relevance dimensions recency and authority. The authority relevance dimension, interacts negatively with both other criteria. Furthermore, despite its importance as a relevance criterion in Twitter (Chen et al., 2012), the authority criterion does not appear to be a factor for the topic, which explain, the negative capacities assigned to $\mu_{(T o, A u)}$ and $\mu_{(R e, A u)}$. However, the higher fuzzy measure associated with $\{T o, R e\}$ indicates a positive interaction between both criteria. Interestingly, all the capacities obtained on the combination of relevance dimensions support the assumption that these criteria usually interact, and this fact should be considered whenever it comes to aggregating them. All these results are consistent with those obtained from the correlation analysis presented in the Correlation Analysis of the Relevance Dimensions section.

\section{Effectiveness Evaluation}

In this section, we report the comparative effectiveness of iAggregator with state-of-the-art aggregation approaches and learning-to-rank methods. 
TABLE 8. Comparative evaluation of retrieval effectiveness with state-of-the-art aggregation operators.

\begin{tabular}{lrrrrr}
\hline \multicolumn{5}{c}{ Precision } \\
\cline { 2 - 5 } Operator & \multicolumn{1}{c}{$\mathrm{P@10}$} & $\mathrm{P} @ 20$ & $\mathrm{P} @ 30$ & \multicolumn{1}{c}{ MAP } & \multirow{2}{*}{ \% Change } \\
\cline { 2 - 5 } Am & $.1140^{*}$ & $.0991^{\dagger}$ & $.0936^{\dagger}$ & .0535 & $\mathbf{+ 5 9 . 8 9 \%}$ \\
Wam & $.1161^{\dagger}$ & $.0991^{\dagger}$ & $.0929^{\dagger}$ & .0539 & $\mathbf{+ 6 0 . 2 8 \%}$ \\
Lcs & $.1860^{*}$ & $.1833^{*}$ & $.1854^{*}$ & .0928 & $\mathbf{+ 2 0 . 7 3 \%}$ \\
Max & $.1088^{\dagger}$ & $.0895^{\dagger}$ & $.0860^{\dagger}$ & .0604 & $\mathbf{+ 6 3 , 2 3 \%}$ \\
Min & $.1793^{*}$ & $.1767^{*}$ & $.1764^{\dagger}$ & .0879 & $\mathbf{+ 2 4 . 5 8 \%}$ \\
Owa & $.1879^{\dagger}$ & $.1776^{*}$ & $.1764^{\dagger}$ & .0882 & $\mathbf{+ 2 4 . 5 8 \%}$ \\
OWMin & $.1897^{\dagger}$ & $.1776^{*}$ & $.1833^{\dagger}$ & .0902 & $\mathbf{+ 2 1 . 6 3 \%}$ \\
And & $.1793^{*}$ & $.1767^{*}$ & $.1764^{\dagger}$ & .0882 & $\mathbf{+ 2 4 . 5 8 \%}$ \\
Scoring & $.2018^{*}$ & $.1982^{*}$ & $.1977^{\dagger}$ & .1091 & $\mathbf{+ 1 5 . 4 7 \%}$ \\
iAggregator & $\mathbf{. 2 3 4 5}$ & $\mathbf{. 2 2 9 3}$ & $\mathbf{. 2 3 3 9}$ & $\mathbf{. 1 2 5 2}$ & \\
& $\mathbf{1 1 3 . 9 4 \%}$ & $\mathbf{+ 1 3 . 5 6 \%}$ & $\mathbf{+ 1 5 . 4 7 \%}$ & $\mathbf{+ 1 2 . 8 5 \%}$ &
\end{tabular}

Note. \% change indicates the iAggregator improvements in terms of P@30. The last row shows the iAggregator improvement in terms of P@X and MAP with the best baseline (i.e., Scoring).

The symbols *, $\dagger$, and $\ddagger$ denote the Student test significance: $* .01<t \leq .05$, ${ }^{\dagger} t \leq .01,{ }^{\ddagger} .05<t \leq .1$.

Comparative evaluation with state-of-the-art aggregation operators. In this section, we compare our approach with some traditional and state-of-the-art aggregation operators, more particularly with the Am, the Wam, and the Lcs, as well as the Min, Max, Owa (Yager, 1988), OWmin (Dubois \& Prade, 1996), And, and Scoring aggregation operators (Costa Pereira et al., 2012). The final scoring function for linear combination is computed as follows: $\operatorname{LCS}(T)=$ $\sum_{i=1}^{3}\left(\alpha_{i} l c s_{i}(T)\right)$, where $\operatorname{lcs}_{i}(T)$ is the performance score of tweet $T$ on the criterion $c_{i}$, with $i \in$ \{topicality, authority, recency\}. The criteria weights used within Wam and Lcs are tuned during the capacities learning phase within the TREC Microblog 2011 topics. The criteria weights used within Wam and Lcs are tuned during the capacities learning phase and then associated the optimal weights, that is, those giving the best average on P@30 within the TREC Microblog 2011 topics: $\alpha_{\text {recency }}=.23, \alpha_{\text {authority }}=.16$, and $\alpha_{\text {topicality }}=.61$, where $\alpha_{i}$ is the weight of the criterion $c_{i}$.

Table 8 reports the results by means of $\mathrm{P} @ 10, \mathrm{P} @ 30$, and $M A P$ obtained by iAggregator against the aforementioned aggregation baseline operators.

As shown in Table 8, our aggregation model outperforms all baselines in both high precisions and MAP. To evaluate the significance of iAggregator improvement, we conducted a paired two-tailed $t$ test. Significance testing based on the Student $t$-test statistic is computed on the basis of all the tested precision levels. The $p$ values are have marked with the symbols $*$, $\dagger$, and $\ddagger$ statistically significant differences. The positive improvements obtained by our approach were found to be statistically significant with $p$ values between .01 and .05 for Lcs, and with $p<.01$ for the other aggregation operators. From Table 8 we also remark that the performances' improvements are important for the classical aggregation operators. We found performance improve- ments up to P@30 values of about $60.26 \%$ for the Wam and of $63.23 \%$ for the Max operator; then the Am had a similar performance, even enough that there is a slight improvement drop. For the Scoring operator, the significant improvement is less important. As we considered the prioritization scenario $S c_{1}$ : $\{$ topicality $\}>\{$ recency $\}>\{$ authority $\}$, giving the best P@30 average, we can conclude that the obtained difference of performance, in favor of iAggregator, is explained by the interactions existing among the set of criteria, which we involved by means of the fuzzy measures. Thus, the global scores can no longer be biased by dependent criteria. Compared with the And operator, the improvement is significantly better. We also notice that although it is a prioritized aggregation method, the And operator exhibits a low performance when compared with those of the Lcs. The same holds for the Owa operator. This can be explained by the tuning performed for Lcs over the criteria weights, during the learning phase to get the best coefficients for each relevance criterion, against the Owa operator, which primarily focuses on the weights with high values and gives low importance to the smallest weights in the evaluation. Because the idea underlying this type of aggregation is to minimize the impact of small document scores with respect to a given criterion, a low weight can be a serious reason for discounting a document, which leads to a biased global evaluation. Regarding the OWmin operator, the performance improvement is about $20 \%$, which is the same as obtained for the Lcs. This method uses a vector of levels of importance to minimize the impact of low weighted terms on the final documents scoring. Unlike Owa, the OWmin operator uses the minimum instead of the average to compute the global documents' scores. This may explain the low performances of the classical averaging aggregation functions as shown by Table 8 . From this analysis we can conclude that the major reason for the performance drop of the aggregation operators is the bias introduced by documents with respect to some criteria, especially those that are dependent (cf. Correlation Analysis of the Relevance Dimensions section).

To get a more detailed understanding of the effectiveness of iAggregator with respect to the other aggregation approaches, we show in the overall curves, plot in Figure 3, a comparison with the aggregation methods. The difference in P@30 values between our approach, Owa, OWmin, and prioritized aggregation operators is more important compared with standard aggregation schemes. As previously discussed, the lowest P@30 values are for the Am and the Wam operators, as well as the Max aggregation method. For the latter, this is likely due to the fact that the global scores are dominated by the best single scores. Roughly speaking, the most satisfied criterion plays the most important role in determining the overall satisfaction degree of a document, even if this relevance criterion is not important for the user (eg., authority). For the Min and And aggregation operators, the similar obtained results are not predictable, because the former is generally dominated by the worst score, whereas the latter, mainly based on the Min operator, penalizes 


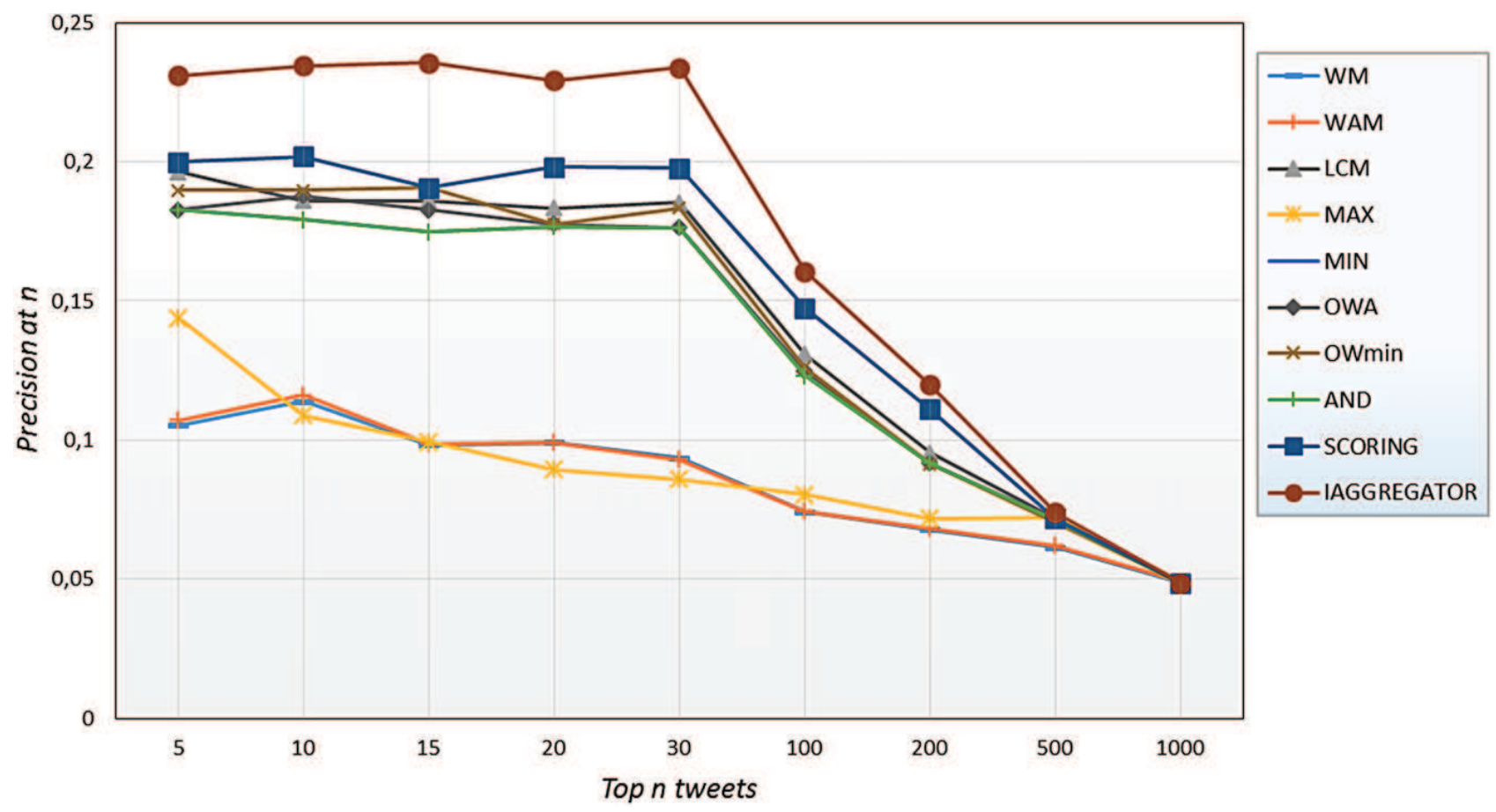

FIG. 3. Average precision at $n$ comparison between iAggregator and standard aggregation mechanisms, as well as some state-of-the-art aggregation operators. [Color figure can be viewed in the online issue, which is available at wileyonlinelibrary.com.]

TABLE 9. Percentage of queries $\mathcal{R}^{+}, \mathcal{R}^{-}$and $\mathcal{R}$ for which iAggregator performs better (lower, equal to) than the different baseline operators, in terms of P@30.

\begin{tabular}{lccccc}
\hline Query set & $A m$ & Min & Owa & Owmin & Scoring \\
\hline $\mathcal{R}^{+}$ & $56.89 \%$ & $43.10 \%$ & $43.10 \%$ & $36.20 \%$ & $36.20 \%$ \\
$\mathcal{R}$ & $22.41 \%$ & $37.93 \%$ & $37.93 \%$ & $43.10 \%$ & $41.37 \%$ \\
$\mathcal{R}^{-}$ & $20.68 \%$ & $18.96 \%$ & $18.96 \%$ & $18.96 \%$ & $22.41 \%$ \\
\hline
\end{tabular}

tweets highly satisfied by the least important criterion. However, if there are many tweets highly scored with respect to the authority criterion (which is likely the case), its overall satisfaction degrees would be biased by this relevance criterion.

To further the effectiveness analysis, we present a gain and failure analysis of the iAggregator approach. Table 9 presents the percentage of queries $\mathcal{R}^{+}, \mathcal{R}^{-}$, and $\mathcal{R}$, for which iAggregator performs better (lower, equal to) than the different baseline operators, in terms of P@30, with an improvement higher (lower, equal to) than $5 \%$ in comparison with the five best baseline operators. From Table 9 we can see that the percentage of queries for which iAggregator is underperformed by the baseline operators is almost the same, with an average of about $20.34 \%$. A manual analysis of these queries revealed that they are practically the same for all the aggregation baselines, with quite a difference for the Am aggregation method. The high percentage for $\mathcal{R}^{+}$ queries is attempted, as expected, for the same aggregation operator, that is, the Am. The difference in percentages is also nearly similar for the three sets of queries, and these latter are almost the same for these three sets with respect to the aforementioned baselines. We note that the lower percentage for $\mathcal{R}^{+}$is marked for the Scoring and OWmin aggregation operators with $36.2 \%$ of queries, whereas for $\mathcal{R}^{-}$ queries, the difference is noticeable for the Scoring operator with a percentage of about $22.41 \%$. For the set of $\mathcal{R}$ queries, because the behavior of iAggregator and the Am aggregation mechanism are totally different, the percentage of queries, for which the performance is in terms of P@30, is equal for both operators and is too low compared with the other baseline operators.

In Figure 4, we plot the difference performances in terms of P@5 ... P@1000 between iAggregator and the best baseline operator, namely, the Scoring operator for both $\mathcal{R}^{+}$ and $\mathcal{R}^{-}$. As shown in Figure 4a, the difference in performance between both aggregation operators is not very significant for queries $\mathcal{R}^{-}$. Despite the fact that the Scoring operator performs well for these queries, our approach is shown to have quite good results. It is notable that our operator gives a null P@30 score for four queries from $\mathcal{R}^{-}$. The average performance difference is about $5.43 \%$, and the high improvement is marked for $n=5$ with a difference equal to $22.21 \%$. The worst P@30 difference performance values are observed for queries $T 63$ and $T 65$ from the set of the TREC Microblog 2012 track topics with the values of $75.01 \%$ and $28.54 \%$, respectively. The first topic, "Bieber and Stewart trading places," is a time-sensitive query. Our model failed in retrieving the most relevant results first. This 


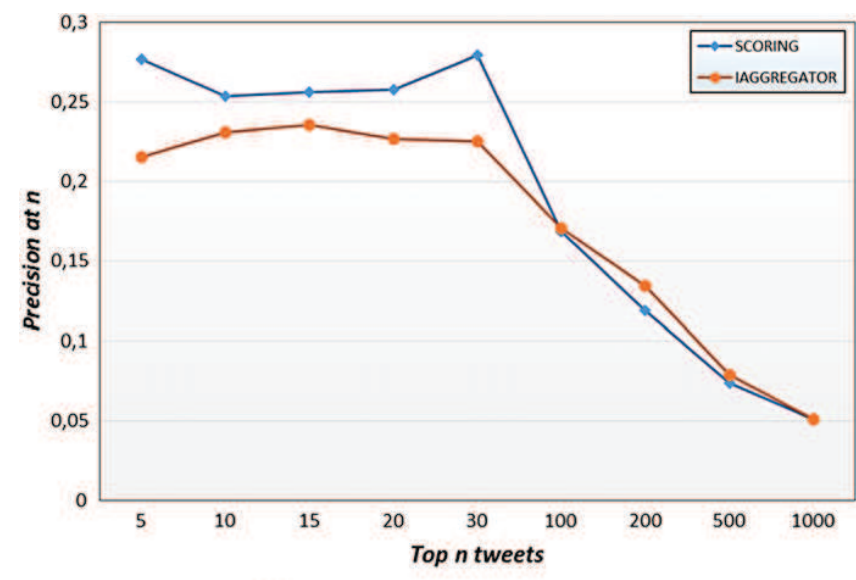

(a) Comparison for queries $\mathcal{R}^{-}$

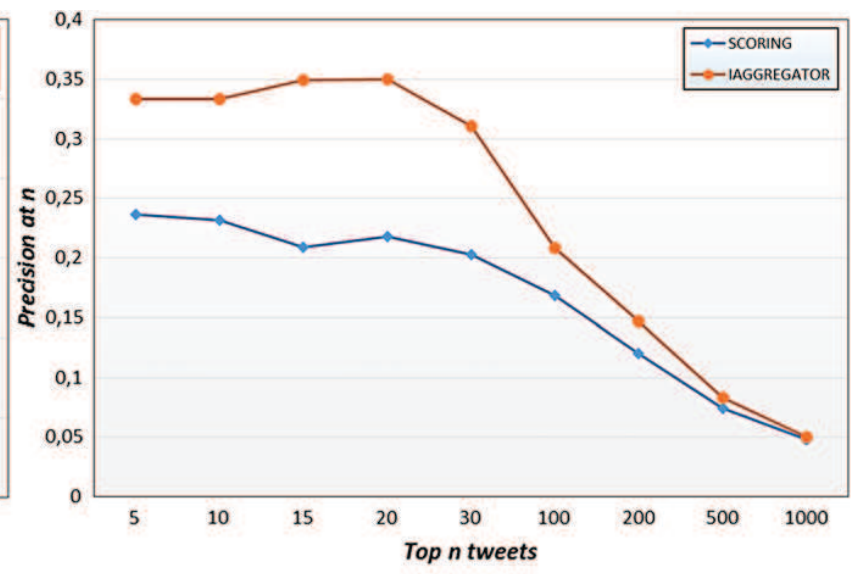

(b) Comparison for queries $\mathcal{R}^{+}$

FIG. 4. Average precision at $n$ comparison between iAggregator and the Scoring aggregation operator for both queries $\mathcal{R}^{-}$and $\mathcal{R}^{+}$. [Color figure can be viewed in the online issue, which is available at wileyonlinelibrary.com.]

is likely due to the low capacity value assigned to the recency criterion $\left(\mu_{R e}=0.204\right)$ compared with the topicality one $\left(\mu_{T o}=0.633\right)$. Although high capacity was assigned to the combination of both relevance dimensions, the Choquet operator failed in retrieving the most relevant tweets on the top of the ranking. The same holds for topic 65, "Michelle Obama's obesity campaign," and this is also likely due to the hypothesis that tweets that are recently published are considered more important. This assumption is not suitable for every topic, because some queries may have relevant tweets that are published in a different prior time without however being recent. For these topics, the Scoring operator performances are quite similar to the other baselines.

For the queries $\mathcal{R}^{+}$, for which iAggregator outperforms the baseline operators, it may be seen from Figure 4 that the performance difference is very significant. This difference is sharper especially for the first top 30 retrieved tweets with an average value of about $34.41 \%$, in contrast with an average value of about 14.10 for $\mathcal{R}^{-}$and for the same retrieved tweets. If we take, for instance, the topic number 73, "Iran nuclear program," we notice that iAggregator performs very well for this one, compared with all the other baselines. Likewise, the iAggregator performance for topic number 56, "Hugo Chavez," is worthwhile compared with the other aggregation operators. These two queries are time sensitive, but unlike topics 65 and 63, they are not relevant only at a given moment in time. More relevant tweets related to these two hot topics are published every day. This may explain the importance given to both relevance criteria (with high capacity values) topicality and recency (cf. Tuning the Choquet Capacities section) after the application of the least-squares-based approach. An in-depth analysis of the nature of topics, as well as the returned relevant tweets, may reveal other interesting issues to improve the accuracy of our aggregation approach.
Comparative evaluation with learning-to-rank methods. We present a comparative evaluation of iAggregator versus conventional state-of-the-art learning-to-rank approaches. More specifically, we test our approach with two pairwise algorithms, RankNet and RankSVM, and with a listwise learning-to-rank algorithm, ListNet. We used the open source code for RankSVM from Joachims (2006) and the RankLib library for the algorithms RankNet and ListNet. ${ }^{7}$ For all the settings, all these algorithms were run for 200 iterations with the measure P@30 as a loss function. For all the settings, all these algorithms were run for 200 iterations with the measure P@30 as a loss function and then trained with the same ground truth used for tuning the best capacity combination (cf. Evaluation Protocol section).

Table 10 shows that iAggregator significantly outperforms both pairwise and listwise algorithms. The improvement is up to 5\% for RankNet and RankSVM, and more than $52 \%$ for the ListNet algorithm. The result for RankSVM is quite lower than the other methods, with an improvement varying between $1.87 \%$ and $5.17 \%$. We also notice that iAggregator enhances the MAP obtained by all the tested approaches with an improvement of $30.43 \%$ for the best baseline RankSVM.

To provide an in-depth understanding of the iAggregator improvement in comparison with its counterparts, we present a gain and failure analysis of the iAggregator approach. Table 11 presents the percentage of queries $\mathcal{R}^{+}$ and $\mathcal{R}^{-}$for which iAggregator performs better (lower) than the different learning-to-rank methods, in terms of P@30.

Clearly, we can see that the percentage of queries for which iAggregator performs better than the learning-to-rank methods is up to $67.24 \%$ for both pairwise algorithms and $72.41 \%$ for the listwise one. Despite the similar percentages

\footnotetext{
${ }^{7}$ http://people.cs.umass.edu/ vdang/ranklib.html
} 
TABLE 10. Comparative evaluation of retrieval effectiveness with conventional learning-to-rank methods.

\begin{tabular}{lrrrrr}
\hline \multicolumn{5}{c}{ Precision } \\
\cline { 2 - 5 } Operator & $\mathrm{P} @ 10$ & $\mathrm{P} @ 20$ & \multicolumn{1}{c}{$\mathrm{P} @ 30$} & \multicolumn{1}{c}{ MAP } & \% change \\
\hline RankSVM & $.2500^{*}$ & $.2250^{\dagger}$ & $.2218^{\dagger}$ & .0871 & $\mathbf{+ 5 . 1 7 \%}$ \\
RankNet & $.2448^{\dagger}$ & $.2198^{\dagger}$ & $.2201^{\dagger}$ & .0858 & $\mathbf{+ 5 . 8 9 \%}$ \\
ListNet & $.0931^{\ddagger}$ & $.1009^{\ddagger}$ & $.1115^{\ddagger}$ & .0485 & $\mathbf{+ 5 2 . 3 3 \%}$ \\
iAggregator & $\mathbf{. 2 3 4 5}$ & $\mathbf{. 2 2 9 3}$ & $\mathbf{. 2 3 3 9}$ & $\mathbf{. 1 2 5 2}$ & \\
& $\mathbf{- 6 . 6 0 \%}$ & $\mathbf{+ 1 . 8 7 \%}$ & $\mathbf{+ 5 . 1 7 \%}$ & $\mathbf{+ 3 0 . 4 3 \%}$ & \\
\hline
\end{tabular}

Note. \% change indicates the iAggregator improvements in terms of P@30. The last row shows the iAggregator improvement in terms of P@X and $M A P$ with the best baseline (i.e., RankSvm).

The symbols *, $\dagger$, and $\ddagger$ denote the Student test significance: *.01<t $\leq .05$; ${ }^{\dagger} .05<t \leq .1 ;{ }^{\ddagger} t \leq .01$

TABLE 11. Percentage of queries $\mathcal{R}^{+}$and $\mathcal{R}^{-}$for which iAggregator performs better (lower) than the different learning-to-rank methods, in terms of P@30.

\begin{tabular}{lccc}
\hline Query set & RankSVM & RankNet & ListNet \\
\hline $\mathcal{R}^{+}$ & $67.24 \%$ & $67.24 \%$ & $72.41 \%$ \\
$\mathcal{R}^{-}$ & $32.76 \%$ & $32.76 \%$ & $27.59 \%$ \\
\hline
\end{tabular}

obtained for $\mathcal{R}^{+}$and $\mathcal{R}^{-}$with respect to RankSVM and RankNet, the analysis of these queries reveals that they are not totally the same for both algorithms. The high percentage for $\mathcal{R}^{+}$queries is achieved with for the ListNet algorithm with a percentage of about $72.41 \%$.

In Figure 5, we plot the difference in performances in terms of P@5 ... P@1000 between iAggregator and RankSVM (the best baseline) for both $\mathcal{R}^{+}$and $\mathcal{R}^{-}$. Obviously, we can note from Figure 5 that the difference of performance between iAggregator and the baseline is quite significant for queries $\mathcal{R}^{-}$. This is not surprising given the fact that the percentage of queries for which iAggregator performs better than RankSVM is relatively high (up to $67.24 \%$ ) and given that the improvement in terms of P@30, despite being significant, is quite low $(+5.17 \%)$.

For the queries $\mathcal{R}^{+}$, for which iAggregator outperforms the baseline learning-to-rank methods, we can see from Figure 5 that the performance difference is less significant. In contrast with $\mathcal{R}^{-}$, for which RankSVM outperforms iAggregator only for the first top 100 tweets, we notice that for $\mathcal{R}^{+}$, RankSVM is outperformed for all the top $K$ tweets. This may explain the high improvement marked by iAggregator in terms of MAP (30.43\%) against the baselines. Likewise, we may further enhance these results by improving the ranking of the relevant tweets returned in the bottom (i.e., below the top 30 tweets).

Comparative evaluation with official TREC Microblog results. We compare our results with the high-performing official results from the TREC Microblog 2012 track (Ounis et al., 2012), in terms of the official measures (P@30 and MAP).

Results shown in Table 12 are rather promising because we outperform the scores of the TREC P@30 and MAP medians. This fact holds despite the quite small number of criteria, which was not the case for most of the participating groups. Moreover, apart from the capacities learning performed over the Microblog 2012 Track topics, we did not make use of any external evidence. It can be seen from Tables 8, 10, and 12 that the MAP values obtained in our IR setting are relatively low compared with those of the official P@30 measure. Because this fact holds for our Choquetbased method, as well as all the tested baselines, we may assert that these low values are not related to the aggregation phase. The major reason for that lies in the rankings returned by the query-likelihood BM25 model (topical criterion), on which were based the computation of the recency and authority document's scores. Still, our results are promising regarding the IR task setting and the track official evaluation measure used to judge the TREC participants' results.

\section{Conclusion}

Aggregation of multiple relevance criteria is attracting increasing attention in the IR community. Research shows performance improvement in the quality of IR systems, when many relevance dimensions are combined together. Prior work reveals that there is a compelling need to design generally effective multicriteria aggregation frameworks to accurately combine all relevance criteria by taking into account their interdependency. In this article, after a critical review of the literature concerning multicriteria relevance aggregation, we proposed a new fuzzy integral-based approach, called iAggregator, based on the Choquet mathematical operator, for multidimensional relevance aggregation. This operator supports the observation that relevance criteria may interact with each other and have a significant effect on how well a ranking is assessed in a real-world IR setting. The effectiveness of the aggregation approach has been evaluated within a social microblogging IR setting, more particularly, a tweet search task where we made use of three relevance criteria. The iAggregator performance evaluation conducted within the TREC Microblog 2011 and 2012 tracks showed that the proposed operator improves the ranking of the documents, in comparison with state-of-theart aggregation operators, when relevance criteria interactions are taken into account by means of the fuzzy measure. An analysis of the success and failure of the search at the query level revealed that our approach performs well for time-sensitive hot topics for which tweets are not only relevant at a given moment in time, and that there is a need to further improve the performed capacity tuning. The study also showed that iAggregator performs better than the other baselines for most of the TREC Microblog 2012 track topics. We also compared our approach with some representative learning-to-rank methods and showed that it performs better in terms of precision at different ranks and MAP. This 


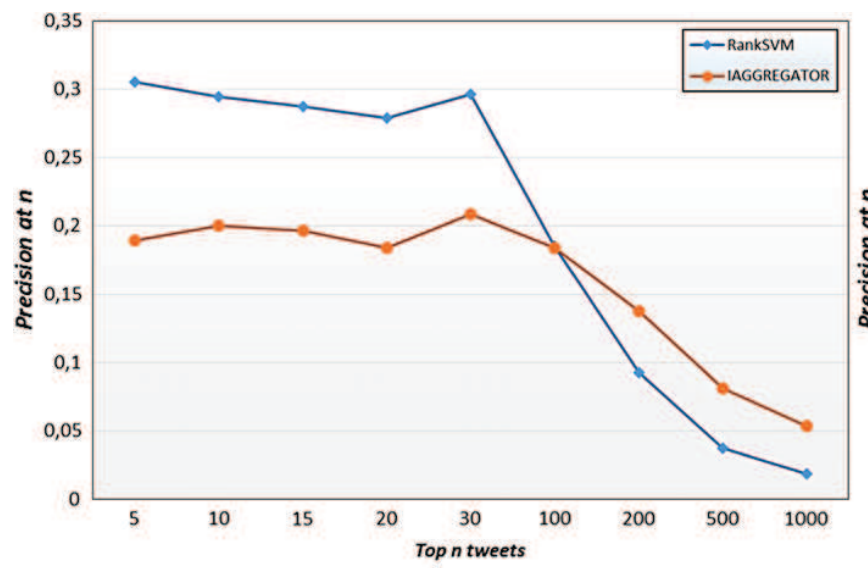

(a) Comparison for queries $\mathcal{R}^{-}$

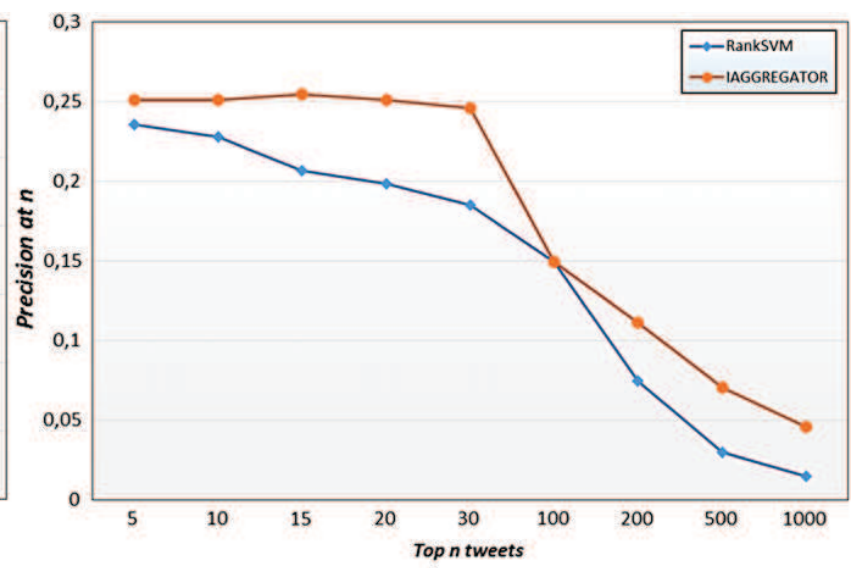

(b) Comparison for queries $\mathcal{R}^{+}$

FIG. 5. Average precision at $n$ comparison between iAggregator and the RankSVM learning-to-rank algorithm for both queries $\mathcal{R}^{-}$and $\mathcal{R}^{+}$. [Color figure can be viewed in the online issue, which is available at wileyonlinelibrary.com.]

TABLE 12. Comparison with the official TREC Microblog 2012 Track results.

\begin{tabular}{lcc}
\hline Model & P@30 & MAP \\
\hline Best 2012 TREC run & .2701 & .2642 \\
Second best run & .2559 & .2277 \\
TREC median & .1808 & .1480 \\
iAggregator & .2339 & .1252 \\
\hline
\end{tabular}

study has some limitations that can be addressed in future work. First, it may be instructive to determine whether the results are generalizable by exploring the evaluation of other retrieval IR settings with a high number of incomparable relevance criteria and then gauge the consistency of the results obtained with those presented in this article. Second, further research is needed to dynamically learn the capacity values through the study of large-scale query profiles; although several works have studied query sensitivity to orthogonal facets (such as navigational, transactional, and informational) (Jansen, Booth, \& Spink, 2008), it would be interesting to shift the study toward multifaceted query sensitivity to dependent criteria and then attempt to tune the user preference criteria, leading to capacity values, along within the user's search sessions. The main outcome of this would be the design of hypotheses supporting optimal tuning of the capacity values considering IR applications where multidimensional relevance is involved.

\section{Acknowledgments}

This work is partially supported by the Franco-Tunisien PHC Utique project $11 G 1417$, entitled EXQUI.

\section{References}

Aczel, J. (1948). On mean values. Bulletin of the American Mathematical Society, 54(4), 392-400.
Ah-Pine, J. (2008). Data fusion in information retrieval using consensus aggregation operators. In Proceedings of the 2008 IEEE/WIC/ACM International Conference on Web Intelligence and Intelligent Agent Technology (Vol. 1, pp. 662-668). Washington, DC: IEEE Computer Society.

Akritidis, L., Katsaros, D., \& Bozanis, P. (2011). Effective rank aggregation for metasearching. Journal of System Software, 84(1), 130-143.

Arrow, K.J. (1974). Choix Collectif et Préférences Individuelles (Vol. 1). I. Aubin (Ed.). Paris, France: Calmann-Lévy.

Aslam, J.A., \& Montague, M. (2001). Models for metasearch. In Proceedings of the 24th Annual International ACM SIGIR Conference on Research and Development in Information Retrieval (pp. 276-284). New York, NY: ACM.

Barry, C.L. (1994). User-defined relevance criteria: An exploratory study. Journal of the American Society for Information Science, 45(3), 149159.

Becker, H., Naaman, M., \& Gravano, L. (2011). Beyond trending topics: Real-world event identification on Twitter. In Proceedings of the Fifth International AAAI Conference on Weblogs and Social Media. Palo Alto, CA: AAAI Press.

Ben Jabeur, L., Tamine, L., \& Boughanem, M. (2010). A social model for literature access: Towards a weighted social network of authors. In Adaptivity, Personalization and Fusion of Heterogeneous Information (pp. 32-39). Paris, France: Le Centre de Hautes Etudes Internationales d'Informatique Documentaire.

Berardi, G., Esuli, A., Marcheggiani, D., \& Sebastiani, F. (2011). ISTI@ TREC microblog track 2011: Exploring the use of hashtag segmentation and text quality ranking. In Proceedings of the 20th Text Retrieval Conference (TREC 2011). Gaithersburg, MD: National Institute of Standards and Technology.

Borlund, P. (2003). The concept of relevance in IR. Journal of the American Society for Information Science and Technology, 54(10), 913925.

Bouidghaghen, O., Tamine, L., Pasi, G., Cabanac, G., Boughanem, M., \& Costa Pereira, C. da. (2011). Prioritized aggregation of multiple context dimensions in mobile IR. In Proceedings of the 7th Asia Conference on Information Retrieval Technology (Vol. 7097, pp. 169-180). Berlin, Heidelberg: Springer.

Box, G.E.P., \& Cox, D.R. (1964). An analysis of transformations. Journal of the Royal Statistical Society, 26(2), 211-252.

Breiman, L., \& Friedman, J.H. (1985). Estimating optimal transformations for multiple regression and correlation. Journal of the American Statistical Association, 80(391), 580-598.

Burges, C., Shaked, T., Renshaw, E., Lazier, A., Deeds, M., Hamilton, N., \& Hullender, G. (2005). Learning to rank using gradient descent. In 
Proceedings of the 22nd International Conference on Machine Learning (pp. 89-96). New York, NY: ACM.

Cantera, J.M., Arias, M., Cabrero, J., Garcia, G., Zubizarreta, A., Vegas, J., $\&$ de la Fuente, P. (2008). MyMoSe: Next generation search engine for mobile users. In Proceedings of the Future of Web Search, the Third Edition of the Future of Web Search Workshop and 2nd CHORUS Conference on Multimedia Retrieval, Soldeu, Andorra.

Cao, Z., Qin, T., Liu, T.-Y., Tsai, M.-F., \& Li, H. (2007). Learning to rank: From pairwise approach to listwise approach. In Proceedings of the 24th International Conference on Machine Learning (pp. 129-136). New York, NY: ACM.

Carterette, B., Kumar, N., Rao, A., \& Zhu, D. (2011). Simple rank-based filtering for microblog retrieval: Implications for evaluation and test collections. In Proceedings of the 20th Text REtrieval Conference (TREC 2011). Gaithersburg, MD: National Institute of Standards and Technology.

Chen, K., Chen, T., Zheng, G., Jin, O., Yao, E., \& Yu, Y. (2012). Collaborative personalized tweet recommendation. In Proceedings of the 35th International ACM SIGIR Conference on Research and Development in Information Retrieval (pp. 661-670). New York, NY: ACM.

Cheverst, K., Davies, N., Mitchell, K., Friday, A., \& Efstratiou, C. (2000). Developing a context-aware electronic tourist guide: Some issues and experiences. In Proceedings of the SIGCHI Conference on Human Factors in Computing Systems (pp. 17-24). New York, NY: ACM.

Choquet, G. (1953). Theory of capacities. Annales de l'Institut Fourier, 5 , 131-295.

Church, K., \& Smyth, B. (2008). Who, what, where \& when: A new approach to mobile search. In Proceedings of the 2008 International Conference on Intelligent User Interfaces (pp. 309-312). New York, NY: ACM.

Condorcet, M. (1785). Essai sur l'Application de l'Analyse à la Probabilité des Décisions Rendues à la Pluralité des Voix. Paris: Imprimerie Royale.

Cong, G., Jensen, C.S., \& Wu, D. (2009). Efficient retrieval of the top-k most relevant spatial web objects. Journal of the Proceedings of the VLDB Endowment, 2, 337-348

Cooper, W.S. (1971). A definition of relevance for information retrieval. Information Storage and Retrieval, 7(1), 19-37.

Cooper, W.S. (1973). On selecting a measure of retrieval effectiveness. Journal of the American Society for Information Science, 24(2), 87-100.

Cosijn, E., \& Ingwersen, P. (2000). Dimensions of relevance. Information Processing \& Management, 36(4), 533-550.

Costa Pereira, C. da, Dragoni, M., \& Pasi, G. (2009). Multidimensional relevance: A new aggregation criterion. In Proceedings of the 31st European Conference on Advances in Information Retrieval (pp. 264-275). Berlin, Heidelberg: Springer.

Costa Pereira, C. da, Dragoni, M., \& Pasi, G. (2012). Multidimensional relevance: Prioritized aggregation in a personalized information retrieval setting. Information Processing \& Management, 48(2), 340-357.

Cuadra, C., \& Katter, R. (1967). Experimental study of relevance judgement (Vol. 1; final report). Cleveland, OH: Case Western Reserve University, School of Library Science, Center for Documentation and Communication Research.

Damak, F., Jabeur, L.B., Cabanac, G., Pinel-Sauvagnat, K., Tamine, L., \& Boughanem, M. (2011). IRIT at TREC microblog 2011. In Proceedings of the 20th Text REtrieval Conference (TREC 2011). Gaithersburg, MD: National Institute of Standards and Technology.

Daoud, M., \& Huang, J.X. (2013). Modeling geographic, temporal, and proximity contexts for improving geotemporal search. Journal of the American Society for Information Science, 64(1), 190-212.

Daoud, M., Tamine, L., \& Boughanem, M. (2010). A personalized graphbased document ranking model using a semantic user profile. In Proceedings of the 18th International Conference on User Modeling, Adaptation, and Personalization (pp. 171-182). Berlin, Heidelberg: Springer-Verlag.

Duan, Y., Jiang, L., Qin, T., Zhou, M., \& Shum, H.-Y. (2010). An empirical study on learning to rank of tweets. In Proceedings of the 23rd International Conference on Computational Linguistics (pp. 295-303). Stroudsburg, PA: Association for Computational Linguistics.
Dubois, D., \& Prade, H. (1996). Semantics of quotient operators in fuzzy relational databases. Fuzzy Sets and Systems, 78, 89-93.

Dubois, D., \& Prade, H. (2004). On the use of aggregation operations in information fusion processes. Fuzzy Sets and Systems, 142(1), 143 161.

Dwork, C., Kumar, R., Naor, M., \& Sivakumar, D. (2001). Rank aggregation methods for the web. In Proceedings of the 10th International Conference on World Wide Web (pp. 613-622). New York, NY: ACM.

Eickhoff, C., de Vries, A.P., \& Collins-Thompson, K. (2013). Copulas for information retrieval. In Proceedings of the 36th Annual International ACM SIGIR Conference on Research and Development in Information Retrieval. Dublin, Ireland: ACM.

Farah, M., \& Vanderpooten, D. (2007). An outranking approach for rank aggregation in information retrieval. In Proceedings of the 30th Annual International ACM SIGIR Conference on Research and Development in Information Retrieval (pp. 591-598). New York, NY: ACM.

Farah, M., \& Vanderpooten, D. (2008). An outranking approach for information retrieval. Information Retrieval, 11(4), 315-334.

Fishburn, P.C. (1972). Mathematics of Decision Theory. The Hague, the Netherlands: Mouton.

Fox, E.A., \& Shaw, J.A. (1993). Combination of multiple searches. In D.K. Harman (Ed.), The 2nd Text REtrieval Conference (TREC2, pp. 243-252). National Institute for Standards and Technology.

Gauch, S., Chaffee, J., \& Pretschner, A. (2003). Ontology-based personalized search and browsing. Web Intelligence and Agent Systems, 1(3-4), 219-234.

Gerani, S., Zhai, C., \& Crestani, F. (2012). Score transformation in linear combination for multi-criteria relevance ranking. In Proceedings of the 34th European Conference on Advances in Information Retrieval (pp. 256-267). Berlin, Heidelberg: Springer-Verlag.

Göker, A., \& Myrhaug, H. (2008). Evaluation of a mobile information system in context. Information Processing \& Management, 44(1), $39-65$.

Grabisch, M. (1995). Fuzzy integral in multicriteria decision making. Fuzzy Sets and Systems, 69(3), 279-298.

Grabisch, M. (1996). The application of fuzzy integrals in multicriteria decision making. European Journal of Operational Research, 89(3), 445456.

Grabisch, M. (2002). Set function over finite sets: Transformations and integrals. In E. Pap (Ed.), Handbook of measure theory (pp. 1381-1401). North Holland: Elsevier.

Grabisch, M., Kojadinovic, I., \& Meyer, P. (2008). A review of methods for capacity identification in choquet integral based multi-attribute utility theory: Applications of the kappalab R package. European Journal of Operational Research, 186(2), 766-785.

Grabisch, M., \& Labreuche, C. (2010). A decade of application of the Choquet and Sugeno integrals in multi-criteria decision aid. Annals of Operations Research, 175(1), 247-286.

Harter, S.P. (1992). Psychological relevance and information science. Journal of the American Society for Information Science, 43(9), 602615.

Hattori, S., Tezuka, T., \& Tanaka, K. (2007). Context-aware query refinement for mobile web search. In Proceedings of the 2007 International Symposium on Applications and the Internet Workshops. Washington, DC: IEEE Computer Society.

Hawking, D., Craswell, N., Bailey, P., \& Griffiths, K. (2001). Measuring search engine quality. Information Retrieval, 4(1), 33-59.

Hwang, C.-L., \& Yoon, K. (1981). Multiple attribute decision making. methods and applications: A state-of-the art survey. Berlin and New York: Springer-Verlag.

James, S. (2010). Use of aggregation functions in decision making (Unpublished doctoral dissertation). School of Information Technology, Faculty of Science and Technology, Deakin University, Melbourne, Australia. Retrieved from http://trove.nla.gov.au/work/168487579

Jankowski, P. (1995). Integrating geographical information systems and multiple criteria decision-making methods. International Journal of Geographical Information Systems, 9(3), 251-273. 
Jansen, B.J., Booth, D.L., \& Spink, A. (2008). Determining the informational, navigational, and transactional intent of web queries. Information Processing \& Management, 44(3), 1251-1266.

Joachims, T. (2006). Training linear svms in linear time. In Proceedings of the 12th ACM SIGKDD International Conference on Knowledge Discovery and Data Mining (pp. 217-226). New York, NY: ACM.

Keeney, R.L., \& Raiffa, H. (1993). Decisions with multiple objectives: Preferences and value tradeoffs (Vol. 42, No. 3). Cambridge, England: Cambridge University Press.

Kendall, M.G. (1938). A new measure of rank correlation. Biometrika, 30(1), 81-93

Kishida, K. (2010). Vocabulary-based re-ranking for geographic and temporal searching at NTCIR geotime task. In Proceedings of the 8th NTCIR Workshop Meeting on Evaluation of Information Access Technologies (pp. 181-184). Tokyo, Japan: National Institute of Informatics.

Kolmogorov, A.N. (1930). On mean values. Rendiconti Accademia dei Lincei, 12(4), 388-391.

Larkey, L.S., Connell, M.E., \& Callan, J. (2000). Collection selection and results merging with topically organized U.S. patents and TREC data. In Proceedings of the Ninth International Conference on Information and Knowledge Management (pp. 282-289). New York, NY: ACM.

Le Calvè, A., \& Savoy, J. (2000). Database merging strategy based on logistic regression. Information Processing \& Management, 36(3), 341359.

Leung, C.W.-K., Chan, S.C.-F., \& Chung, F.-L. (2006). A collaborative filtering framework based on fuzzy association rules and multiple-level similarity. Knowledge and Information Systems, 10(3), 357-381.

Liang, F., Qiang, R., Hong, Y., Fei, Y., \& Yang, J. (2012). PKUICST at TREC 2012 microblog track. In Proceedings of the 21th Text REtrieval Conference (TREC 2012). Gaithersburg, MD: National Institute of Standards and Technology.

Liu, F., Yu, C., \& Meng, W. (2004). Personalized web search for improving retrieval effectiveness. IEEE Transactions on Knowledge and Data Engineering, 16(1), 28-40.

Liu, T.-Y. (2009). Learning to rank for information retrieval. Foundations and Trends in Information Retrieval, 3(3), 225-331.

Ma, Z., Pant, G., \& Sheng, O.R.L. (2007). Interest-based personalized search. ACM Transactions on Information Systems, 25(1), 5.

Macdonald, C., Santos, R., \& Ounis, I. (2013). The whens and hows of learning to rank for web search. Information Retrieval, 16, 584 628.

Mata, F., \& Claramunt, C. (2011). Geost: Geographic, thematic and temporal information retrieval from heterogeneous web data sources. In Proceedings of the 10th International Conference on Web and Wireless Geographical Information Systems (pp. 5-20). Berlin, Heidelberg: Springer-Verlag.

Menger, K. (1942). Statistical metrics. Proceedings of the National Academy of Sciences of the United States of America, 28(12), 535537.

Metzler, D., \& Cai, C. (2011). USC/ISI at TREC 2011: Microblog track. In Proceedings of the 20th Text REtrieval Conference (TREC 2011). Gaithersburg, MD: National Institute of Standards and Technology.

Miyanishi, T., Seki, K., \& Uehara, K. (2012). TREC 2012 microblog track experiments at kobe university. In Proceedings of the 21th Text REtrieval Conference (TREC 2012). Gaithersburg, MD: National Institute of Standards and Technology.

Mizzaro, S. (1998). How many relevances in information retrieval? Interacting with Computers, 10(3), 303-320.

Murofushi, T., \& Soneda, S. (1993). Techniques for reading fuzzy measures (iii): Interaction index. In Proceedings of the 9th Fuzzy Systems Symposium, Sapporo, Japan (pp. 693-696). New York: Institute of Electrical and Electronics Engineers (IEEE)

Nagmoti, R., Teredesai, A., \& De Cock, M. (2010). Ranking approaches for microblog search. In Proceedings of the 2010 IEEE/WIC/ACM International Conference on Web Intelligence and Intelligent Agent Technology (Vol. 01, pp. 153-157). Washington, DC: IEEE Computer Society.

Neumann, J., \& Morgenstern, O. (1953). Theory of Games and Economic Behavior (3rd ed.). Princeton, NJ: Princeton University Press.
Ounis, T., Macdonald, J., \& Soboroff, I. (2011). Overview of the TREC2011 microblog track. In Proceedings of the 20th Text REtrieval Conference (TREC 2011). Gaithersburg, MD: National Institute of Standards and Technology.

Ounis, T., Macdonald, J., \& Soboroff, I. (2012). Overview of the TREC2012 microblog track. In Proceedings of the 21th Text REtrieval Conference (TREC 2012). Gaithersburg, MD: National Institute of Standards and Technology.

Palacio, D., Cabanac, G., Sallaberry, C., \& Hubert, G. (2010). On the evaluation of geographic information retrieval systems: Evaluation framework and case study. International Journal on Digital Libraries, 11(2), 91-109.

Rees, A., \& Schultz, D. (1967). A field experiment approach to the study of relevance assessments in relation to document searching (Vol. 2). Cleveland, OH: Center for Documentation and Communication Research, School of Library Science, Case Western Reserve University.

Renda, M.E., \& Straccia, U. (2003). Web metasearch: Rank vs. score based rank aggregation methods. In Proceedings of the 2003 ACM Symposium on Applied Computing (pp. 841-846). New York, NY: ACM.

Saracevic, T. (1996). Relevance reconsidered. In Ingwersen, P., \& Pors, N.O. (Eds.), Second International Conference on Conceptions in Library and Information Science: Integration in Perspective (pp. 201-218). Copenhagen, Denmark: Royal School of Librarianship.

Saracevic, T. (2000). Digital library evaluation: Toward evolution of concepts. Library Trends, 49(3), 350-369.

Saracevic, T. (2007). Relevance: A review of the literature and a framework for thinking on the notion in information science. Part III: Behavior and effects of relevance. Journal of the American Society for Information Science, 58(13), 2126-2144.

Saracevic, T., Rothenberg, D., \& Stephan, P. (1974). Study of information utility. Proceedings of the American Society for Information Science, 11, 234-238.

Schamber, L. (1991). Users' criteria for evaluation in a multimedia environment. Proceedings of the 54th ASIS Annual Meeting, 28, 126133

Schilit, B.N., LaMarca, A., Borriello, G., Griswold, W.G., McDonald, D., Lazowska, E., Iverson, W. (2003). Challenge: Ubiquitous location-aware computing and the "place lab" initiative. In Proceedings of the 1st ACM International Workshop on Wireless Mobile Applications and Services on WLAN Hotspots (pp. 29-35). New York, NY: ACM.

Schweizer, B., \& Sklar, A. (1960). Statistical metrics. Pacific Journal of Mathematics, 10(1), 313-334.

Schweizer, B., \& Sklar, A. (1983). Probabilistic Metric Spaces. New York: North-Holland Publishing Co.

Shapley, L.S. (1953). A value for n-person games. In H.W. Kuhn \& A.W. Tucker (Eds.), Contributions to the theory of games (Vol. 28, pp. 307317). Princeton, NJ: Princeton University Press.

Si, L., \& Callan, J. (2002). Using sampled data and regression to merge search engine results. In Proceedings of the 25th Annual International ACM SIGIR Conference on Research and Development in Information Retrieval (pp. 19-26). New York, NY: ACM

Sieg, A., Mobasher, B., \& Burke, R. (2007). Web search personalization with ontological user profiles. In Proceedings of the Sixteenth ACM Conference on Information and Knowledge Management (pp. 525-534). New York, NY: ACM.

Smith, M., Barash, V., Getoor, L., \& Lauw, H.W. (2008). Leveraging social context for searching social media. In Proceedings of the 2008 ACM Workshop on Search in Social Media (pp. 91-94). New York, NY: ACM.

Steuer, R.E. (1986). Multiple Criteria Optimization: Theory, Computation and Application. New York, NY: John Wiley \& Sons.

$\mathrm{Su}$, L.T. (1992). Evaluation measures for interactive information retrieval. Information Processing \& Management, 28(4), 503-516.

$\mathrm{Su}$, L.T. (1994). The relevance of recall and precision in user evaluation. Journal of the American Society for Information Science, 45(3), 207-217.

Taylor, A.R. (2012). User relevance criteria choices and the information search process. Information Processing \& Management, 48(1), 136153. 
Taylor, A.R., Cool, C., Belkin, N.J., \& Amadio, W.J. (2007). Relationships between categories of relevance criteria and stage in task completion. Information Processing \& Management, 43(4), 1071-1084.

Taylor, R.S. (1986). Value-Added Processes in Information Systems. Melvin J. Voigt (Ed.). Greenwood Publishing Group Inc., Westport, CT, USA.

Torra, V. (2005). Aggregation operators and models. Fuzzy Sets and Systems, 156(3), 407-410.

Triantaphyllou, E. (2000). Multi-criteria decision making methods. In Multi-criteria Decision Making Methods: A Comparative Study (Vol. 44, pp. 5-21). New York: Springer US.

Vickery, B.C. (1959). Subject analysis for information retrieval. In Proceedings of the International Conference on Scientific Information (Vol. 2, pp. 855-865). Washington, DC: The National Academies Press.

Vogt, C.C., \& Cottrell, G.W. (1999). Fusion via a linear combination of scores. Information Retrieval, 1(3), 151-173.

Wei, F., Li, W., \& Liu, S. (2010). iRANK: A rank-learn-combine framework for unsupervised ensemble ranking. Journal of the American Society for Information Science and Technology, 61(6), 1232-1243.
Wei, Z., Zhou, L., Li, B., Wong, K.-F., Gao, W., \& Wong, K.-F. (2011). Exploring tweets normalization and query time sensitivity for twitter search. In Proceedings of the 20th Text REtrieval Conference (TREC 2011). National Institute of Standards and Technology (NIST).

Wolfe, S.R., \& Zhang, Y. (2010). Interaction and personalization of criteria in recommender systems. In Proceedings of the 18th International Conference on User Modeling, Adaptation, and Personalization (pp. 183194). Berlin, Heidelberg: Springer-Verlag.

Yager, R.R. (1988). On ordered weighted averaging aggregation operators in multicriteria decision making. IEEE Transactions on Systems Man and Cybernetics, 18(1), 183-190.

Yau, S.S., Liu, H., Huang, D., \& Yao, Y. (2003). Situation-aware personalized information retrieval for mobile internet. In Proceedings of the 27th Annual International Conference on Computer Software and Applications (p. 638). Washington, DC: IEEE Computer Society. 